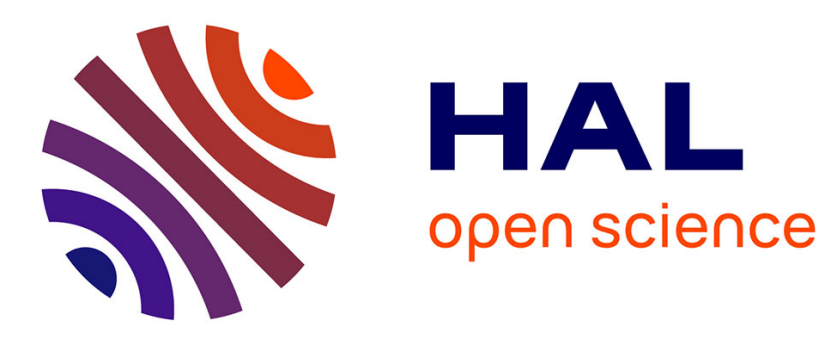

\title{
Mesures quadratiques de la proximité des diviseurs
}

\author{
A Raouj, A Stef, G Tenenbaum
}

\section{To cite this version:}

A Raouj, A Stef, G Tenenbaum. Mesures quadratiques de la proximité des diviseurs. Mathematical Proceedings of the Cambridge Philosophical Society, 2011, 150 (1), pp.73-96. 10.1017/S0305004110000411 . hal-01280600

\section{HAL Id: hal-01280600 https://hal.science/hal-01280600}

Submitted on 1 Mar 2016

HAL is a multi-disciplinary open access archive for the deposit and dissemination of scientific research documents, whether they are published or not. The documents may come from teaching and research institutions in France or abroad, or from public or private research centers.
L'archive ouverte pluridisciplinaire HAL, est destinée au dépôt et à la diffusion de documents scientifiques de niveau recherche, publiés ou non, émanant des établissements d'enseignement et de recherche français ou étrangers, des laboratoires publics ou privés. 


\title{
Mesures quadratiques de la proximité des diviseurs
}

\author{
A. Raouj, A. Stef \& G. Tenenbaum
}

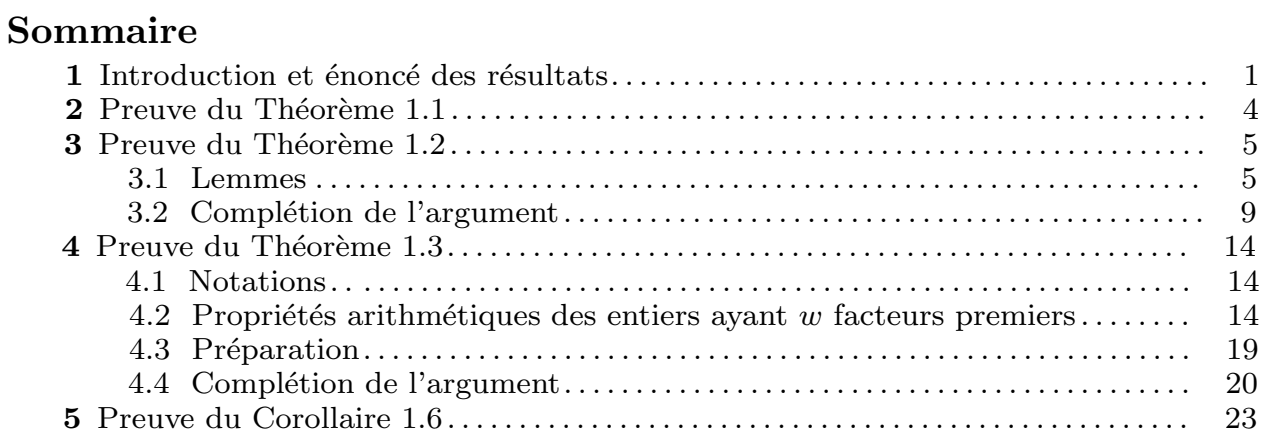

\section{Introduction et énoncé des résultats}

L'aspect fractal de la suite des diviseurs d'un entier normal peut être révélé de diverses manières, comme l'existence d'une dimension de Hausdorff convenablement définie [5]. Cependant, les critères les plus fins sont liés à la conjecture d'Erdős datant de la fin des années 1930, mentionnée dans [3] et établie dans [6], selon laquelle presque tout entier possède deux diviseurs dont le rapport est dans l'intervalle $] 1,2[$. Plusieurs mesures quantitatives associées à cette conjecture ont été considérées dans [4], notamment les fonctions arithmétiques

$$
E(n):=\min _{d d^{\prime} \mid n, d<d^{\prime}} \log \left(d^{\prime} / d\right), \quad U(n, \alpha):=\sum_{\begin{array}{c}
d d^{\prime} \mid n,\left(d, d^{\prime}\right)=1 \\
\left|\log \left(d^{\prime} / d\right)\right| \leqslant(\log n)^{\alpha}
\end{array}} 1 \quad(\alpha \in \mathbb{R}) .
$$

D'intéressantes variantes de ces fonctions sont étudiées dans [10] et [15].

Comme dans [4], définissons deux nouvelles fonctions arithmétiques $n \mapsto e(n)$ et $n \mapsto u(n, \alpha)$ par les relations

$$
\begin{aligned}
E(n) & =:(\log n)^{1-\log 3} \exp \left\{e(n) \sqrt{\log _{2} n}\right\}, \\
U(n, \alpha) & =:(\log n)^{\log 3-1+\alpha} \exp \left\{u(n, \alpha) \sqrt{\log _{2} n}\right\}, \quad(n \geqslant 3) .
\end{aligned}
$$

Ici et dans la suite, nous désignons par $\log _{k}$ la $k$-ième itérée de la fonction logarithme. Il a été établi dans [4] que l'on a

$$
e(n) \ll \sqrt{\log _{3} n}, \quad u(n, \alpha) \ll \sqrt{\log _{3} n} \quad \mathrm{pp},
$$

où la mention pp indique que la relation ainsi désignée est valable sur un ensemble d'entiers de densité naturelle unité. Une hypothèse heuristique d'équirépartition des quantités $\log \left(d^{\prime} / d\right)$ lorsque $d d^{\prime} \mid n$ conduit à supposer que l'on a, en un sens à préciser,

$$
E(n) \approx \frac{\log n}{3^{\omega(n)}}, \quad U(n, \alpha) \approx 3^{\omega(n)}(\log n)^{\alpha-1} \quad \mathrm{pp},
$$

où $\omega(n)$ désigne le nombre des facteurs premiers distincts d'un entier $n$. Il est en particulier conjecturé dans [4], chap. 5, p. 98, que les fonctions $e(n)$ et $u(n, \alpha)$ possèdent des lois de répartition limites. L'un des objectifs du présent travail consiste à établir cette conjecture. 
Nous notons $\Phi$ la fonction de Gauss

$$
\Phi(y):=\frac{1}{\sqrt{2 \pi}} \int_{-\infty}^{y} \mathrm{e}^{-t^{2} / 2} \mathrm{~d} t \quad(y \in \mathbb{R}) .
$$

Théorème 1.1. Les fonctions arithmétiques $n \mapsto e(n)$ et $n \mapsto u(n, \alpha)(\alpha>1-\log 3)$ possèdent des lois de répartition limites dont la fonction de répartition commune $F$ est définie par $F(y):=\Phi(y / \log 3)$. Plus précisément, pour tout $\alpha>1-\log 3$ fixé et uniformément sous les conditions $x>16, y \in \mathbb{R}$, nous avons

$$
\sum_{\substack{n \leqslant x \\ e(n) \leqslant y}} 1=x F(y)+O\left(\frac{x\left(\log _{3} x\right)^{2}}{\sqrt{\log _{2} x}}\right), \quad \sum_{\substack{n \leqslant x \\ u(n, \alpha) \leqslant y}} 1=x F(y)+O\left(\frac{x\left(\log _{3} x\right)^{2}}{\sqrt{\log _{2} x}}\right) .
$$

De plus, la loi de répartition de la fonction $n \mapsto u(n, 1-\log 3)$ est donnée par

$$
F^{*}(y):= \begin{cases}0 & \text { si } y<0 \\ \Phi(y / \log 3) & \text { si } y \geqslant 0\end{cases}
$$

et, lorsque $\alpha=1-\log 3$, la seconde relation (1·1) a lieu uniformément pour $x>16, y \geqslant 0$, quitte à remplacer $F$ par $F^{*}$.

Remarque. Lorsque $\alpha<1-\log 3$, on a $U(n, \alpha)=1 \mathrm{pp}$; la question de la répartition de $u(n, \alpha)$ est donc sans objet.

Pour tout entier $n \geqslant 1$ et tout réel $t \geqslant 0$, nous posons

$$
\nabla(n, t)=\nabla^{-}(n, t):=\sum_{\substack{d d^{\prime}\left|n,\left(d, d^{\prime}\right)=1\\\right| \log \left(d^{\prime} / d\right) \mid \leqslant t}} 1, \quad \nabla^{+}(n, t):=1+\sum_{\substack{d d^{\prime}|n \\ 0<| \log \left(d^{\prime} / d\right) \mid \leqslant t}} 1,
$$

de sorte que

$$
E(n)=\min \left\{t \geqslant 0: \nabla^{ \pm}(n, t)>1\right\}, \quad U(n, \alpha)=\nabla\left(n,(\log n)^{\alpha}\right) \quad(n \geqslant 1) .
$$

Notre approche consiste à évaluer $\nabla^{ \pm}(n, t)$ sur les ensembles

$$
\mathcal{E}_{w}(x):=\{n \leqslant x: \omega(n)=w\}
$$

lorsque le paramètre entier $w$ est voisin de l'ordre normal $\log _{2} x$.

Nous posons

$$
\pi_{w}(x):=\left|\mathcal{E}_{w}(x)\right| \quad(x \geqslant 1) .
$$

Sauf mention contraire, dans toute la suite de ce travail, les constantes impliquées par les symboles $\ll$ de Vinogradov et $O$ de Landau sont absolues et la lettre $c$, avec ou sans indice, désigne une constante absolue strictement positive.

Théorème 1.2. Pour une constante convenable $c>0$ et sous les conditions

$$
x>16, \quad t \geqslant 0, \quad w \in \mathbb{N}, \quad\left|w / \log _{2} x-1\right| \leqslant c, \quad \frac{6}{5} \log _{3} x \leqslant \xi \leqslant \frac{1}{110} \log _{2} x,
$$

l'inégalité

$$
\nabla^{+}(n, t) \leqslant 1+\frac{t 3^{w} w \mathrm{e}^{3 \xi}}{\log x}
$$

a lieu pour tous les entiers $n$ de $\mathcal{E}_{w}(x)$ sauf au plus $\ll \pi_{w}(x) \mathrm{e}^{-\xi / 244}$ exceptions. 
Théorème 1.3. Soit $B>0$. Il existe une constante $c_{1}>0$ telle que, sous les conditions

$$
\begin{aligned}
& x>16, \quad 0 \leqslant t \leqslant \log x, \quad\left(\log _{2} x\right)^{10} \leqslant \psi \leqslant(\log x)^{B}, \\
& w \in \mathbb{N}, \quad\left|w-\log _{2} x\right| \leqslant(\log \psi)^{1 / 4}\left(\log _{2} x\right)^{1 / 2},
\end{aligned}
$$

l'inégalité

$$
\nabla^{-}(n, t) \geqslant \frac{3^{w} t}{\psi \log x}
$$

ait lieu pour tous les entiers $n$ de $\mathcal{E}_{w}(x)$ sauf au plus $\ll \mathrm{e}^{-c_{1} \sqrt{\log \psi}} \pi_{w}(x)$ exceptions.

Désignons par $\Omega(n)$ le nombre des facteurs premiers d'un entier $n$, comptés avec multiplicité, et posons

$$
Q(y):=y \log y-y+1 \quad(y>0)
$$

Une version quantitative classique de l'inégalité de Hardy-Ramanujan — voir par exemple [16], formule (3.52) p. 438 - stipule que, pour tous $\varepsilon \in] 0, \frac{1}{2}[, x>3$, on a

$$
(1-\varepsilon) \log _{2} x \leqslant \omega(n) \leqslant \Omega(n) \leqslant(1+\varepsilon) \log _{2} x
$$

pour tous les entiers $n \leqslant x$ sauf au plus $\ll \varepsilon^{-2} x(\log x)^{-Q(1+\varepsilon)}$ exceptions. Cela nous permet de déduire des Théorèmes $1.2\left(\operatorname{avec} \xi:=\frac{4}{3} \log _{3} x\right)$ et $1.3\left(\right.$ avec $\left.\psi:=\left(\log _{2} x\right)^{10}\right)$ le corollaire suivant, établi grâce aux formules $(1 \cdot 3)$.

Corollaire 1.4. (i) Pour chaque $\alpha \in]-\infty, 1]$ fixé et tout $x>16$, les inégalités

$$
E(n) \geqslant \frac{\log n}{3^{\omega(n)}\left(\log _{2} n\right)^{5}}, \quad U(n, \alpha) \leqslant 1+3^{\omega(n)}\left(\log _{2} n\right)^{5}(\log n)^{\alpha-1}
$$

ont lieu pour tous les entiers $n \leqslant x$ sauf au plus $\ll x\left(\log _{2} x\right)^{-1 / 183}$ exceptions.

(ii) Il existe une constante $b_{1}>0$ telles que, pour chaque $\left.\left.\alpha \in\right]-\infty, 1\right]$ fixé et tout $x>16$, les inégalités

$$
E(n) \leqslant \frac{2 \log n}{3^{\omega(n)}}\left(\log _{2} n\right)^{10}, \quad U(n, \alpha) \geqslant \frac{3^{\omega(n)}(\log n)^{\alpha-1}}{2\left(\log _{2} n\right)^{10}}
$$

aient lieu pour tous les entiers $n \leqslant x$ sauf au plus $\ll x \mathrm{e}^{-b_{1} \sqrt{\log _{3} x}}$ exceptions.

Nous pouvons également exploiter les renseignements acquis sur la taille des ensembles exceptionnels pour obtenir une évaluation uniforme, valable pp, de la fonction $t \mapsto \nabla(n, t)$.

Corollaire 1.5. Il existe une constante absolue $A>0$ telle que

$$
\frac{3^{\omega(n)} t}{\log n} \mathrm{e}^{-A\left(\log _{3} n\right)^{2}} \leqslant \nabla^{ \pm}(n, t) \leqslant 1+\frac{t 3^{\omega(n)}\left(\log _{2} n\right)^{736}}{\log n} \quad(0 \leqslant t \leqslant \log n) \quad \text { pp. }
$$

Démonstration. Il suffit d'appliquer les Théorèmes 1.2 et 1.3 avec

$$
\xi:=245 \log _{3} x, \quad \psi:=\mathrm{e}^{2\left(\log _{3} x\right)^{2} / c_{1}^{2}},
$$

aux points tests $t_{j}:=\mathrm{e}^{j}\left(-\log _{2} x-1 \leqslant j \leqslant 1+\log _{2} x\right)$. Le résultat annoncé découle alors de la croissance en $t$ de $\nabla(n, t)$. 
Ce résultat permet à son tour l'estimation de fonctions arithmétiques générales du type

$$
\vartheta(n ; f):=\sum_{\substack{d d^{\prime} \mid n \\ d<d^{\prime}}} f\left(\log \left(d^{\prime} / d\right)\right) .
$$

Nous dirons qu'une fonction $f:] 0, \infty[\rightarrow] 0, \infty[$ est à croissance polynomiale s'il existe une constante $K(f)>0$ telle que

$$
f(a) / b^{K(f)} \leqslant f(a b) \leqslant f(a) b^{K(f)} \quad(a>0, b \geqslant 1) .
$$

Corollaire 1.6. Soit $f:] 0, \infty[\rightarrow] 0, \infty[$ une fonction à croissance polynomiale. Nous avons

$$
\vartheta(n ; f)=\frac{3^{\omega(n)}}{\log n} \mathrm{e}^{O\left(\left(\log _{3} n\right)^{2}\right)} \int_{(\log n) / 3^{\omega(n)}}^{\log n} f(t) \mathrm{d} t \quad \text { pp. }
$$

Le cas $f(t):=1 / t^{\alpha}(\alpha>0)$ de (1.11) est particulièrement intéressant. Nous obtenons

$$
\sum_{\substack{d d^{\prime} \mid n \\ d<d^{\prime}}} \frac{1}{\left\{\log \left(d^{\prime} / d\right)\right\}^{\alpha}}=\frac{3^{\omega(n)}+3^{\alpha \omega(n)}}{(\log n)^{\alpha}} \mathrm{e}^{O\left(\left(\log _{3} n\right)^{2}\right)} \quad \mathrm{pp},
$$

ce qui indique notamment que la sommation est essentiellement dominée par son plus grand terme si, et seulement si, $\alpha \geqslant 1$.

\section{Preuve du Théorème 1.1}

Le Théorème 1.1 découle du Corollaire 1.4 - mais on pourrait également utiliser une variante effective, établie de la même manière, du Corollaire 1.5 - par le biais du théorème classique d'Erdős-Kac, dont nous rappelons ci-dessous un énoncé avec terme d'erreur optimal. ${ }^{(1)}$

Lemme 2.1 (Erdős-Kac). Nous avons, uniformément pour $x \geqslant 3$ et $y \in \mathbb{R}$,

$$
\left|\left\{n \leqslant x: \frac{\omega(n)-\log _{2} x}{\sqrt{\log _{2} x}} \leqslant y\right\}\right|=x \Phi(y)+O\left(\frac{x}{\sqrt{\log _{2} x}}\right) .
$$

Cela permet de prouver très simplement le Théorème 1.1. Nous nous contentons de fournir les détails dans le cas de la fonction $e(n)$, les calculs étant essentiellement identiques pour $u(n, \alpha)$.

Il résulte des Théorèmes 1.2 et 1.3 que l'on a, pour une constante $A$ convenable,

$$
\frac{\log n}{3^{\omega(n)}\left(\log _{2} n\right)^{A}} \leqslant E(n) \leqslant \frac{\log n}{3^{\omega(n)}} \mathrm{e}^{A\left(\log _{3} n\right)^{2}}
$$

pour tous les entiers $n \leqslant x$ sauf au plus $\ll x / \log _{2} x$ exceptions. En reportant dans la définition, nous obtenons, pour ces mêmes entiers,

$$
e(n)=\frac{(\log 3)\left\{\log _{2} n-\omega(n)\right\}}{\sqrt{\log _{2} n}}+O\left(\frac{\left(\log _{3} n\right)^{2}}{\sqrt{\log _{2} n}}\right) .
$$

Au vu de $(2 \cdot 1)$, cela implique immédiatement le résultat annoncé.

1. Voir, par exemple, [16] p. 474 


\section{Preuve du Théorème 1.2}

\subsection{Lemmes}

Le résultat suivant, établi dans [14], fournit une borne supérieure, génériquement exacte à une constante multiplicative près, pour la valeur moyenne sur $\varepsilon_{w}(x)$ d'une fonction multiplicative positive ou nulle vérifiant une condition de croissance peu restrictive.

Ici et dans toute la suite, la lettre $p$ désigne exclusivement un nombre premier.

Lemme 3.1. Soient $A$ et $B$ deux nombres réels $>0$ et $f$ une fonction arithmétique multiplicative à valeurs dans $[0,+\infty[$ telle que

$$
\sum_{p^{\nu} \leqslant x} f\left(p^{\nu}\right) \log p^{\nu} \leqslant A x \quad(x \geqslant 2), \quad \sum_{p} \sum_{\nu \geqslant 2} \frac{f\left(p^{\nu}\right)}{p^{\nu}} \leqslant B .
$$

Pour $w \geqslant 1, x \geqslant 2$, nous avons

$$
\sum_{n \in \mathcal{E}_{w}(x)} f(n) \ll \frac{A x}{(w-1) ! \log x}\left(\sum_{p \leqslant x} \frac{f(p)}{p}+B\right)^{w-1},
$$

où la constante implicite est absolue. En particulier, pour toute constante $R>0$, et uniformément sous la condition $1 \leqslant w \leqslant R \log _{2} x$, nous avons

$$
\sum_{n \in \mathcal{E}_{w}(x)} f(n) \ll \pi_{w}(x) \exp \left\{\varrho \sum_{p \leqslant x} \frac{f(p)-1}{p}\right\}
$$

où l'on a posé $\varrho:=(w-1) / \log _{2} x$ et où la constante implicite dépend au plus de $A, B, R$.

Nous ferons un usage essentiel du résultat suivant, relatif au nombre des entiers $n$ sans petit facteur premier et pour lesquels $\omega(n)$ est fixé. Nous notons $P^{-}(n)$ le plus petit facteur premier d'un entier naturel $n$ avec la convention $P^{-}(1)=\infty$. Pour $x \geqslant y>1$ et $k \in \mathbb{N}^{*}$, nous posons

$$
\pi_{k}(x, y)=\left|\left\{n \leqslant x: \omega(n)=k, P^{-}(n)>y\right\}\right| .
$$

Lemme 3.2. Soit $R>0$. Il existe $u_{0}=u_{0}(R)>0$ tel que l'on ait uniformément pour $u:=(\log x) / \log y \geqslant u_{0}(R), 1 \leqslant k \leqslant R \log u$,

$$
\pi_{k}(x, y) \asymp \frac{x}{\log x} \frac{(\log u)^{k-1}}{(k-1) !} .
$$

Démonstration. L'estimation indiquée est une conséquence très affaiblie de formules asymptotiques dues à Balazard [2] (lemme 5) et Alladi [1] (théorème 7). Alladi montre que l'on a uniformément pour $\exp \left\{\left(\log _{2} x\right)^{3}\right\} \leqslant y \leqslant \sqrt{x}$ et $1 \leqslant k \leqslant R \log u$,

$$
\pi_{k}(x, y)=\frac{\mathrm{e}^{-\gamma \xi}}{\Gamma(1+\xi)} \frac{x}{\log x} \frac{(\log u)^{k-1}}{(k-1) !}\left\{1+O_{R}\left(\frac{1}{\sqrt{\log u}}\right)\right\}
$$

avec $\xi:=k /(\log u-\gamma){ }^{(2)}$ Balazard, en employant d'ailleurs de manière essentielle d'autres formules établies par Alladi dans [1], montre que

$$
\pi_{k}(x, y)=g(\varrho, y) \frac{x}{\log x} \frac{(\log u)^{k-1}}{(k-1) !}\left\{1+O_{R}\left(\frac{1}{\log _{2} x}\right)\right\}
$$

2. Ici, nous désignons par $\Gamma$ la fonction Gamma d'Euler et par $\gamma$ la constante d'Euler. 
uniformément pour $\frac{3}{2} \leqslant y \leqslant \exp \left\{(\log x)^{2 / 5}\right\}, 1 \leqslant k \leqslant R \log u$, avec $\varrho:=(k-1) / \log u$ et

$$
g(\varrho, y):=\frac{(\log y)^{\varrho}}{\Gamma(\varrho+1)} \prod_{p \leqslant y}\left(1-\frac{1}{p}\right)^{\varrho} \prod_{p>y}\left(1-\frac{1}{p}\right)^{\varrho}\left(1+\frac{\varrho}{p-1}\right) .
$$

La relation $(3 \cdot 2)$ résulte immédiatement de $(3 \cdot 3)$ et $(3 \cdot 4)$. Nous omettons les détails de la vérification.

Lemme 3.3. Sous les conditions $k \in \mathbb{N}^{*}, x \geqslant y>\exp \left\{(\log k)^{2}\right\}, u:=(\log x) / \log y$, nous avons uniformément

$$
\pi_{k}(x, y) \ll \frac{x}{\log x} \frac{(\log u)^{k-1} \sqrt{k}}{(k-1) !}
$$

Démonstration. Considérons d'abord le cas $k \leqslant 2 \log u$. Nous avons alors

$$
\pi_{k}(x, y) \ll \frac{x}{\log x} \frac{(\log u)^{k-1}}{(k-1) !} .
$$

Cela résulte du Lemme 3.2 si $u>u_{0}(2)$. Si $u \leqslant u_{0}(2)$, alors $k$ est également borné et $(3 \cdot 6)$ résulte par exemple du crible de Brun.

Plaçons-nous donc dans le cas $k>2 \log u$. Pour tout $z \geqslant 1$, nous pouvons écrire, notant $\mu$ la fonction de Möbius,

$$
\begin{aligned}
\pi_{k}(x, y) & \leqslant z^{-k} \sum_{\substack{m n \leqslant x \\
P^{-}(m n)>y}} \mu(m)^{2}(z-1)^{\omega(m)} \\
& \ll \frac{x}{z^{k} \log y} \prod_{y<p \leqslant x}\left(1+\frac{z-1}{p}\right)+\frac{1}{z^{k}} \sum_{\substack{x / y<m \leqslant x \\
P^{-}(m)>y}} \mu(m)^{2}(z-1)^{\omega(m)} \\
& \ll \frac{x(1+z / u)}{z^{k} \log y} \prod_{y<p \leqslant x}\left(1+\frac{z-1}{p}\right) \\
& \ll \frac{x(1+z / u)}{z^{k} \log y} \exp \left\{(z-1) \log u+O\left(z \mathrm{e}^{-\sqrt{\log y}}\right)\right\},
\end{aligned}
$$

où l'avant-dernière majoration a été obtenue par application du th. 01 de [4]. Cela implique immédiatement le résultat si $k=1$, en choisissant par exemple $z=1$. Si $k \geqslant 2$, nous choisissons $z:=k / \log u>2$ en tenant compte du fait que $u \geqslant k$. Nous obtenons

$$
\pi_{k}(x, y) \ll \frac{x\{(\log u) / k\}^{k} \mathrm{e}^{k-\log u}}{\log y} \asymp \frac{x}{\log x} \frac{(\log u)^{k} \sqrt{k}}{k !} \ll \frac{x}{\log x} \frac{(\log u)^{k-1} \sqrt{k}}{(k-1) !} .
$$

Les deux lemmes suivants concernent la répartition des facteurs premiers des entiers de $\mathcal{E}_{w}(x)$. Nous désignons par $\left\{p_{k}(n): 1 \leqslant k \leqslant \omega(n)\right\}$ la suite croissante des facteurs premiers distincts d'un entier générique $n$ et posons

$$
\omega(n, t):=\sum_{\substack{p^{\nu} \| n \\ p \leqslant t}} 1, \quad \Omega(n, t):=\sum_{\substack{p^{\nu} \| n \\ p \leqslant t}} \nu \quad\left(n \in \mathbb{N}^{*}, t>0\right) .
$$


Lemme 3.4. Soit $R>1$. Sous les conditions

$$
\begin{array}{r}
x>16, \quad 3 \leqslant \xi \leqslant x, \quad w \in \mathbb{N}, \quad \frac{1}{R} \log _{2} x \leqslant w \leqslant R \log _{2} x, \\
\varrho:=(w-1) / \log _{2} x, \quad 1<\alpha \leqslant 2, \quad \beta:=\varrho Q(1 / \alpha),
\end{array}
$$

les inégalités

$$
\begin{aligned}
& \max _{\xi<t \leqslant x}\left\{\omega(n, t)-\alpha \varrho \log _{2} t\right\} \leqslant 0, \\
& \min _{\xi<t \leqslant x}\left\{\omega(n, t)-\frac{\varrho}{\alpha} \log _{2} t\right\} \geqslant 0,
\end{aligned}
$$

ont lieu pour tous les entiers $n$ de $\mathcal{E}_{w}(x)$ sauf au plus $\ll_{R} \beta^{-1}(\log \xi)^{-\beta} \pi_{w}(x)$ exceptions.

Si nous supposons de plus $\alpha \leqslant \alpha_{0}<2$, les mêmes estimations sont valables pour $\Omega(n, t)$ à la place de $\omega(n, t)$.

Démonstration. Contentons-nous de considérer (3.7), le cas de (3.8) et de la fonction $\Omega(n, t)$ relevant de manipulations identiques. Pour $j \in \mathcal{J}:=\left[\log _{2} \xi-1, \log _{2} x+1\right] \cap \mathbb{N}$, posons $t_{j}:=\exp \exp j$. Notant $E$ l'ensemble des entiers $n$ de $\mathcal{E}_{w}(x)$ tels que

$$
\max _{j \in \mathcal{J}}\left\{\omega\left(n, t_{j}\right)-\alpha \varrho(j-1)\right\}>0,
$$

nous avons, pour tout $y, 1 \leqslant y \leqslant 2$,

$$
|E| \leqslant \sum_{j \in \mathcal{J}} \sum_{n \in \mathcal{E}_{w}(x)} y^{\omega\left(n, t_{j}\right)-\alpha \varrho(j-1)} \ll_{R} y^{\alpha \varrho} \pi_{w}(x) \sum_{j \in \mathcal{J}} y^{-\alpha \varrho j} \mathrm{e}^{\varrho(y-1) j},
$$

où la dernière majoration résulte du Lemme 3.1. En choisissant $y:=\alpha \in] 1,2]$, nous obtenons

$$
|E| \ll \beta_{1}^{-1}(\log \xi)^{-\beta_{1}} \pi_{w}(x),
$$

avec $\beta_{1}:=\varrho Q(\alpha)>\beta$.

Considérons à présent un entier $n \in \mathcal{E}_{w}(x) \backslash E$. Pour tout $t$ de $\left.] \xi, x\right]$, il existe un unique indice $j \in \mathcal{J}$ tel que $t_{j}<t \leqslant t_{j+1}$. Nous avons alors

$$
\omega(n, t) \leqslant \omega\left(n, t_{j+1}\right) \leqslant \alpha \varrho \log _{2} t_{j} \leqslant \alpha \varrho \log _{2} t,
$$

donc $n$ satisfait $(3 \cdot 7)$.

Lemme 3.5. Soit $R>1$. Sous les conditions

$$
\begin{array}{r}
x>16, \quad 3 \leqslant \xi \leqslant \log _{2} x, \quad w \in \mathbb{N}, \quad \frac{1}{R} \log _{2} x \leqslant w \leqslant R \log _{2} x, \\
\varrho:=(w-1) / \log _{2} x, \quad 1<\alpha \leqslant \frac{3}{2}, \quad \beta:=Q(\alpha),
\end{array}
$$

les inégalités

$$
\begin{aligned}
& \max _{1 \leqslant k \leqslant w}\left\{\varrho \log \left(\frac{\log x}{\log p_{k}(n)}\right)-\alpha(w-k)\right\} \leqslant \xi \\
& \min _{1 \leqslant k \leqslant w}\left\{\varrho \log \left(\frac{\log x}{\log p_{k}(n)}\right)-\frac{w-k}{\alpha}\right\} \geqslant-\xi
\end{aligned}
$$

ont lieu pour tous les entiers $n$ de $\mathcal{E}_{w}(x)$ sauf au plus $\ll_{R} \beta^{-1} \mathrm{e}^{-\beta \xi} \pi_{w}(x)$ exceptions. 
Démonstration. Commençons par évaluer le nombre des exceptions à (3·10). Le résultat annoncé étant trivialement vérifié si $\xi \leqslant 2 R$, nous pouvons supposer dans la suite $\xi>2 R$. L'expression entre accolades dans $(3 \cdot 10)$ vaut

$$
(1-\alpha) w-1+\alpha k-\varrho \log _{2} p_{k}(n) .
$$

Elle est donc au plus égale à $\xi$ lorsque $\alpha k \leqslant(\alpha-1) w+\frac{3}{4} \xi \leqslant(\alpha-1) w+\xi+\varrho \log _{2} 2$. Dans le cas contraire, elle est évidemment majorée par $y_{k}-\varrho \log _{2} p_{k}(n)$, avec

$$
y_{k}:=k-\frac{3}{4}(\alpha-1)(w-k) \geqslant \frac{(\alpha-1) w}{4 \alpha}+\frac{3(3 \alpha+1) \xi}{16 \alpha}>\frac{2}{3} \xi .
$$

De plus, pour tout $n$ de $\varepsilon_{w}(x)$, l'inégalité

$$
y_{k}-\varrho \log _{2} p_{k}(n) \geqslant \frac{2}{3} \xi
$$

équivaut à $\omega(n)-\omega\left(n, z_{k}\right) \leqslant w-k$ où l'on a posé $z_{k}:=\exp \exp \left\{\left(y_{k}-\frac{2}{3} \xi\right) / \varrho\right\}$.

Désignons par $W_{k}$ le nombre des entiers $n$ de $\mathcal{E}_{w}(x)$ vérifiant $(3 \cdot 12)$, de sorte que $\sum_{1 \leqslant k \leqslant w} W_{k}$ représente une majoration du nombre des exceptions à (3·10). Pour tout $v \in] 0,1]$, nous avons

$$
\begin{aligned}
W_{k} & \leqslant \sum_{n \in \mathcal{E}_{w}(x)} v^{\omega(n)-\omega\left(n, z_{k}\right)+k-w} \ll \pi_{w}(x) \exp \left\{(k-w) \log v+\varrho(v-1)\left(\log _{2} x-\log _{2} z_{k}\right)\right\} \\
& \ll \pi_{w}(x) \exp \left\{-(w-k) \log v+(v-1)\left(\frac{1}{4}(3 \alpha+1)(w-k)+\frac{2}{3} \xi\right)\right\},
\end{aligned}
$$

en vertu du Lemme 3.1. Choisissant $v:=4 /\{3 \alpha+1\}$, nous obtenons

$$
W_{k} \ll \pi_{w}(x) \mathrm{e}^{-Q(v)(w-k) / v-\frac{2}{3}(1-v) \xi} .
$$

On déduit le résultat annoncé de cette estimation par sommation sur $k \leqslant w$ en observant que $\frac{2}{3}(1-v)=2(\alpha-1) /(3 \alpha+1)>Q(\alpha)$ pour $1<\alpha \leqslant \frac{3}{2}$.

Des manipulations similaires permettent d'estimer le nombre des entiers $n$ de $\mathcal{E}_{w}(x)$ contrevenant à (3.11). L'étape liminaire consiste à se ramener, trivialement, au cas $k \leqslant w-\alpha \xi$. Il suffit ensuite d'observer que tout entier $n$ contrevenant à (3·11) vérifie

$$
\omega(n)-\omega\left(n, x_{k}\right) \geqslant w-k
$$

avec $x_{k}:=\exp \exp \{(w-(w-k) / \alpha+\xi-1) / \varrho\}$. On peut donc majorer le nombre des entiers exceptionnels par

$$
\sum_{k \leqslant w-\alpha \xi} \sum_{n \in \mathcal{E}_{w}(x)} \alpha^{\omega(n)-\omega\left(n, x_{k}\right)-w+k} \ll \pi_{w}(x) Q(\alpha)^{-1} \mathrm{e}^{-Q(\alpha) \xi} .
$$

Introduisons, pour chaque entier $n$ de $\varepsilon_{w}(x)$, les produits

$$
n_{[k]}:=\prod_{1 \leqslant j \leqslant k} p_{j}(n)^{\nu_{j}(n)} \quad(0 \leqslant k \leqslant w)
$$

où $\nu_{j}(n)$ désigne l'exposant de $p_{j}(n)$ dans la décomposition canonique de $n$. Notre dernier lemme concerne le comportement normal des facteurs premiers multiples des entiers de $\mathcal{E}_{w}(x)$. Nous considérons en particulier la fonction de Piltz

$$
\tau_{3}(n):=\sum_{d d^{\prime} \mid n} 1=\prod_{p^{\nu} \| n}\left(\begin{array}{c}
\nu+2 \\
2
\end{array}\right) \quad(n \geqslant 1) .
$$


Lemme 3.6. Soit $R>1$. Sous les conditions

$$
x>16, \quad w \in \mathbb{N}, \quad \frac{1}{R} \log _{2} x \leqslant w \leqslant R \log _{2} x, \quad T \geqslant 1,
$$

nous avons

$$
\sup _{1 \leqslant k \leqslant w} \tau_{3}\left(n_{[k]}\right) / 3^{k} \leqslant T
$$

pour tous les entiers $n$ de $\mathcal{E}_{w}(x)$ sauf au plus $\ll \pi_{w}(x) / T$. De plus, la relation

$$
\exists k \in[2 \xi, w]: p_{k}(n)^{2} \mid n
$$

est réalisée pour au plus $\ll \mathrm{e}^{-Q\left(\frac{1}{2}\right) \xi} \pi_{w}(x)$ entiers de $\mathcal{E}_{w}(x)$.

Démonstration. Nous avons $\tau_{3}\left(n_{[k]}\right) / 3^{k} \leqslant \tau_{3}(n) / 3^{w}=\tau_{3}(n) / 3^{\omega(n)}$ pour tout indice $k$. Or

$$
\sum_{n \in \mathcal{E}_{w}(x)} \frac{\tau_{3}(n)}{3^{\omega(n)}} \ll \pi_{w}(x)
$$

d'après le Lemme 3.1. Cela établit la première assertion de l'énoncé. La seconde résulte du Lemme 3.4 avec par exemple $\alpha:=2$ : le nombre des entiers exceptionnels est

$$
\ll \pi_{w}(x) \mathrm{e}^{-Q\left(\frac{1}{2}\right) \xi}+\sum_{\nu \geqslant 2} \sum_{p>\exp \exp (\xi / R)} \pi_{w-1}\left(\frac{x}{p^{\nu}}\right) \ll \pi_{w}(x)\left(\mathrm{e}^{-Q\left(\frac{1}{2}\right) \xi}+\exp \left\{-\mathrm{e}^{\xi / R}\right\}\right) .
$$

\subsection{Complétion de l'argument}

La constante $c$ étant supposée assez petite et les paramètres $w$, $\xi$, vérifiant

$$
w \in \mathbb{N}, \quad(1-c) \log _{2} x \leqslant w \leqslant(1+c) \log _{2} x, \quad \frac{6}{5} \log _{3} x \leqslant \xi \leqslant \frac{1}{110} \log _{2} x,
$$

nous posons

$$
\vartheta^{-}:=\frac{\log x}{2 \mathrm{e}^{3 \xi} 3^{w} w}, \quad \vartheta^{+}:=\mathrm{e}^{-2 \xi} \log x .
$$

Il suffit d'établir (1.4) lorsque $\vartheta^{-} \leqslant t \leqslant \vartheta^{+}$. Lorsque $t>\vartheta^{+}$, l'inégalité est impliquée par la majoration triviale $\nabla^{+}(n, t) \leqslant \tau_{3}(n)$ au vu du Lemme 3.6. Lorsque $t<\vartheta^{-}$, le résultat appliqué à $\vartheta^{-}$fournit $\nabla^{+}(n, t) \leqslant \nabla^{+}\left(n, \vartheta^{-}\right)=1$ avec la majoration requise pour le nombre des exceptions. Dans toute la suite de ce paragraphe, nous supposons donc

$$
\vartheta^{-} \leqslant t \leqslant \vartheta^{+}
$$

Soit $\left.\alpha:=\frac{78}{71} \in\right] 1, \log 3[$. Pour chaque valeur du paramètre entier $\xi$, nous considérons le sous-ensemble $\mathcal{E}_{w}^{*}(x)$ de $\mathcal{E}_{w}(x)$ défini par les inégalités $(3 \cdot 10),(3 \cdot 11)$ et les conditions

$$
\min _{2 \xi<k \leqslant w} \log _{2} p_{k}(n) / k>1 /(\varrho \alpha), \max _{1 \leqslant k \leqslant w} \tau_{3}\left(n_{[k]}\right) / 3^{k} \leqslant(\mathrm{e} / 2)^{\xi}, p_{k}(n)^{2} \mid n \Rightarrow k \leqslant 2 \xi .
$$

D'après les Lemmes $3.4,3.5$ et 3.6 , nous avons

$$
\pi_{w}^{*}(x):=\left|\mathcal{E}_{w}^{*}(x)\right| \geqslant\left\{1-B \mathrm{e}^{-\beta \xi}\right\} \pi_{w}(x)
$$

où $\beta:=\min \left\{Q\left(\frac{1}{2}\right), \frac{99}{100} Q(1 / \alpha), Q(\alpha)\right\}$ et $B$ est une constante convenable. Notons que $\beta \geqslant \frac{1}{244}$. 
Posons

$$
V_{t}(m, z):=\sum_{\substack{d d^{\prime}|m \\ 0<| \log \left(d^{\prime} / d\right)-z \mid \leqslant t}} 1 \quad\left(m \in \mathbb{N}^{*}, z \in \mathbb{R}\right),
$$

de sorte que $\nabla^{+}(m, t)=1+V_{t}(m, 0)$ pour tout entier $m$. Notons que

$$
V_{t}(m n, z) \leqslant \sum_{d d^{\prime} \mid m} V_{t}\left(n, z+\log \left(d^{\prime} / d\right)\right) \quad(m \geqslant 1, n \geqslant 1, z \in \mathbb{R})
$$

avec égalité si $(m, n)=1$.

Notre méthode consiste à évaluer $V_{t}\left(n_{[k]}, 0\right)$ par récurrence sur $k, 2 \xi<k \leqslant w$, en exploitant la relation

$$
V_{t}\left(n_{[k+1]}, 0\right)=V_{t}\left(n_{[k]}, 0\right)+2 V_{t}\left(n_{[k]}, \log p_{k+1}(n)\right) \quad(2 \xi<k<w) .
$$

Plus précisément, nous employons une méthode d'espérance conditionnelle analogue à celle qui a été utilisée dans [13], [7] et [8] pour majorer les moments de la fonction Delta de Hooley, et nous sommons la relation $(3 \cdot 21)$ sur l'ensemble de tous les entiers de $\mathcal{E}_{w}^{*}(x)$ tels que $n_{[k]} / n_{[2 \xi]}$ est fixé. Spécifiquement, posant

$$
\begin{aligned}
& \mathcal{A}_{k}:=\left\{a: \exists n \in \mathcal{E}_{w}^{*}(x), n_{[k]} / n_{[2 \xi]}=a\right\} \quad(2 \xi<k<w), \\
& \mathcal{M}_{\xi}(k, a):=\left\{m: \exists n \in \mathcal{E}_{w}^{*}(x), n_{[k]}=m a\right\} \quad\left(2 \xi<k<w, a \in \mathcal{A}_{k}\right) \text {, } \\
& \mathcal{M}_{\xi}:=\bigcup_{2 \xi<k<w} \bigcup_{a \in \mathcal{A}_{k}} \mathcal{M}_{\xi}(k, a),
\end{aligned}
$$

nous pouvons écrire, pour $a \in \mathcal{A}_{k}, 2 \xi<k \leqslant w-2$, compte tenu de (3·17),

$$
\begin{aligned}
S_{k+1}(a) & :=\sum_{\substack{n \in \mathcal{E}_{w}^{*}(x) \\
n_{[k]} / n_{[2 \xi]}=a}}\left\{V_{t}\left(n_{[k+1]}, 0\right)-V_{t}\left(n_{[k]}, 0\right)\right\} \log p_{k+1}(n) \\
\leqslant & \sum_{\substack{m \in \mathcal{M}_{\xi}(k, a) \\
P^{+}(m)<P^{-}(a) \\
P^{+}(a)<p<\sqrt{x / a m}}}\left\{V_{t}(\text { map }, 0)-V_{t}(m a, 0)\right\}(\log p) \pi_{w-k-1}\left(\frac{x}{m a p}, p\right),
\end{aligned}
$$

où, ici et dans la suite, $P^{+}(m)$ désigne le plus grand facteur premier de l'entier $m$ avec la convention $P^{+}(1)=1$. La première des conditions $(3 \cdot 17)$ garantit que l'on a, dans la dernière sommation,

$$
\log _{2} p>2 \xi / \varrho \alpha>2 \log _{2}\{1+w-k\} .
$$

Nous pouvons donc employer le Lemme 3.3 pour majorer $\pi_{w-k-1}(x /$ map, $p)$, soit

$$
\pi_{w-k-1}\left(\frac{x}{m a p}, p\right) \ll \frac{x \sqrt{w}}{m a p \log (x / m a)} \frac{\{\log u(m a)\}^{w-k-2}}{(w-k-2) !},
$$

où l'on a posé

$$
u(m a):=\frac{\log (x / m a)}{\log P^{+}(a)} \geqslant \frac{\log (x / m a p)}{\log p} .
$$

La minoration de $p_{w}(n)$ contenue dans $(3 \cdot 10)$ implique $\log (x / m a)>\mathrm{e}^{-\xi / \varrho} \log x$ pour tous $a \in \mathcal{A}_{k}, m \in \mathcal{M}_{\xi}(k, a)$. Par $(3 \cdot 21)$, nous obtenons donc

$$
S_{k+1}(a) \ll \sum_{\substack{m \in \mathcal{M}_{\xi}(k, a) \\ P^{+}(m)<P^{-}(a)}} \frac{x \sqrt{w} \mathrm{e}^{\xi / \varrho}}{m a \log x} \frac{\{\log u(a m)\}^{w-k-2}}{(w-k-2) !} Y_{t}(m a)
$$

avec

$$
Y_{t}(m a):=\sum_{p>P^{+}(a)} \frac{V_{t}(m a, \log p) \log p}{p} .
$$


En exprimant $V_{t}(m a, \log p)$ comme une somme sur les diviseurs de $m a$ et en intervertissant les sommations, nous obtenons

$$
Y_{t}(m a) \leqslant \sum_{d d^{\prime} \mid m a} \sum_{\substack{p>P^{+}(a) \\-t-\log \left(d^{\prime} / d\right) \leqslant \log p \leqslant t-\log \left(d^{\prime} / d\right)}} \frac{\log p}{p} .
$$

Notons que $\log P^{+}(a)>\mathrm{e}^{k / \alpha \varrho}$ en vertu de $(3 \cdot 17)$. D'après le théorème des nombres premiers, la somme intérieure de $(3 \cdot 25)$ vaut donc

$$
2 t+O\left(\exp \left\{-\mathrm{e}^{k / 2 \alpha \varrho}\right\}\right) \ll t,
$$

où la dernière majoration est justifiée par la minoration $k>2 \xi>2 \alpha \varrho \log _{3} x$ dès que, par exemple, $c<\frac{1}{11}$. Il suit

$$
Y_{t}(m a) \ll t \tau_{3}(m a) \leqslant t 3^{k}(\mathrm{e} / 2)^{\xi} .
$$

Reportons dans $(3 \cdot 24)$. Posant $L:=1+1 / \varrho-\log 2$, nous obtenons

$$
S_{k+1}(a) \ll \frac{x \sqrt{w} \mathrm{e}^{L \xi} 3^{k} t}{(w-k-2) ! \log x} \sum_{\substack{m \in \mathcal{M}_{\xi}(k, a) \\ P^{+}(m)<P^{-}(a)}} \frac{\{\log u(m a)\}^{w-k-2}}{m a} .
$$

Notant $P^{+}(a)=P^{+}(m a)=p, b=m a / p$, avec donc $\omega(b)=k-1$, nous avons

$$
\begin{aligned}
\sum_{\substack{a \in \mathcal{A}_{k}, m \in \mathcal{M}_{\xi}(k, a) \\
P^{+}(m)<P^{-}(a)}} \frac{\{\log u(m a)\}^{w-k-2}}{m a(w-k-2) !} & \leqslant \sum_{p \leqslant x} \sum_{\substack{\omega(b)=k-1 \\
k \leqslant \varrho \alpha \log _{2} p \\
P^{+}(b) \leqslant p}} \frac{\left(\log _{2} x-\log _{2} p\right)^{w-k-2}}{p b(w-k-2) !} \\
& \ll \sum_{p \leqslant x} \frac{\left(\log _{2} x-\log _{2} p\right)^{w-k-2}\left(\log _{2} p\right)^{k-1}}{p(k-1) !(w-k-2) !} \\
& \ll \frac{\left(\log _{2} x\right)^{w-2}}{(w-3) !} \ll w \frac{\left(\log _{2} x\right)^{w-1}}{(w-1) !} .
\end{aligned}
$$

En reportant dans $(3 \cdot 26)$, il suit

$$
\sum_{n \in \mathcal{E}_{w}^{*}(x)}\left\{V_{t}\left(n_{[k+1]}, 0\right)-V_{t}\left(n_{[k]}, 0\right)\right\} \log p_{k+1}(n) \ll t 3^{k} w^{3 / 2} \mathrm{e}^{L \xi} \pi_{w}(x),
$$

et donc, en vertu de $(3 \cdot 10)$,

$$
\sum_{n \in \mathcal{E}_{w}^{*}(x)}\left\{V_{t}\left(n_{[k+1]}, 0\right)-V_{t}\left(n_{[k]}, 0\right)\right\} \ll \frac{t 3^{k} w^{3 / 2} \mathrm{e}^{\alpha(w-k) / \varrho+(L+1 / \varrho) \xi}}{\log x} \pi_{w}(x) .
$$

Par sommation sur $k \in] 2 \xi, w-2]$, il suit

$$
\sum_{n \in \mathcal{E}_{w}^{*}(x)}\left\{V_{t}\left(n_{[w-1]}, 0\right)-V_{t}\left(n_{[2 \xi]}, 0\right)\right\} \ll \frac{t 3^{w} w^{3 / 2} \mathrm{e}^{(L+1 / \varrho) \xi}}{\log x} \pi_{w}(x) .
$$

Posant $K:=L+\beta+1 / \varrho=1+\beta+2 / \varrho-\log 2$, nous en déduisons que

$$
V_{t}\left(n_{[w-1]}, 0\right)-V_{t}\left(n_{[2 \xi]}, 0\right) \ll \frac{t 3^{w} w^{3 / 2} \mathrm{e}^{K \xi}}{\log x}
$$

pour tous les entiers de $\mathcal{E}_{w}(x)$ sauf au plus $\ll \pi_{w}(x) \mathrm{e}^{-\beta \xi}$ exceptions. 
En choisissant la constante $c$ de l'énoncé du Théorème 1.2 assez petite, il s'ensuit que la majoration

$$
\nabla^{+}\left(n_{[w-1]}, t\right) \leqslant \nabla^{+}\left(n_{[2 \xi]}, t\right)+\frac{t 3^{w} w \mathrm{e}^{3 \xi}}{4 \log x}
$$

a lieu, pour tout $t \in\left[\vartheta^{-}, \vartheta^{+}\right]$et tous les entiers $n$ de $\mathcal{E}_{w}(x)$ sauf au plus $\ll \pi_{w}(x) \mathrm{e}^{-\beta \xi}$.

Soit alors $z_{0}:=\exp \exp \{4 \xi /(1-c)\}$. D'après $(3 \cdot 8)$ avec $\alpha=2$ et $t=z_{0}$, nous avons $p_{2 \xi}(n) \leqslant z_{0}$ pour tous les entiers $n$ de $\mathcal{E}_{w}(x)$ sauf au plus $\ll \mathrm{e}^{-4 Q(1 / 2) \xi} \pi_{w}(x)$ exceptions. De plus, la relation

$$
\sum_{n \in \mathcal{E}_{w}(x)}\left(\frac{3}{2}\right)^{\Omega(n)-\omega(n)} \ll \pi_{w}(x),
$$

qui découle du Lemme 3.1, fournit, quitte à négliger $\ll(2 / 3)^{\xi} \pi_{w}(x)$ nouveaux entiers de $\mathcal{E}_{w}(x)$, la majoration $n_{[2 \xi]} \leqslant z:=z_{0}^{3 \xi}$.

Nous avons donc $\nabla^{+}\left(n_{[2 \xi]}, t\right) \leqslant 1+\nabla_{0}(n)+2 \nabla_{1}(n)$ avec

$$
\nabla_{0}(n):=\sum_{\substack{d \mid n \\ 1<d \leqslant \mathrm{e}^{t}}} 1, \quad \nabla_{1}(n):=\sum_{\substack{d \mid n \\ d \leqslant z}} \sum_{1<d<d^{\prime} \leqslant \mathrm{e}^{t} d} 1 .
$$

Nous allons montrer que la majoration

$$
\nabla_{j}(n) \ll \frac{t 3^{w}}{\log x} \quad(j=0,1)
$$

a lieu pour tous les entiers restants sauf au plus un nombre acceptable d'exceptions.

Lorsque $j=0$, nous pouvons supposer $t \geqslant \log 2$ puisque $\nabla_{0}(n, t)=0$ dans le cas contraire. Soit $h:=(\log x)^{1 / 10}$. D'après le Lemme 3.4, nous avons

$$
\Omega\left(n, \mathrm{e}^{t+h}\right) \leqslant(1+c) \varrho \log (t+h)
$$

pour tous les entiers $n$ de $\mathcal{E}_{w}(x)$ sauf au plus $\ll \pi_{w}(x) /(\log x)^{B}$ exceptions, où l'on a posé $B:=\frac{1}{10}(1-c) Q(1 /(1+c))$. Cela implique, pour les entiers non exceptionnels,

$$
\nabla_{0}(n) \leqslant 2^{\Omega\left(n, \mathrm{e}^{t}\right)} \leqslant(t+h)^{(1+c)^{2} \log 2} .
$$

Nous en déduisons bien $(3 \cdot 30)$ pour $j=0$ dès que $c$ est assez petite puisque le membre de droite de $(3 \cdot 30)$ excède $t(\log x)^{(1-c) \log 3-1}$.

Pour traiter le cas $j=1$, nous observons que tout couple $\left\{d, d^{\prime}\right\}$ compté dans $\nabla_{1}(n)$ vérifie

$$
t \geqslant \log \left(d^{\prime} / d\right) \geqslant \log \left(d^{\prime} /\left(d^{\prime}-1\right)\right)>1 / d^{\prime}>\mathrm{e}^{-t} / d
$$

et donc $d>\mathrm{e}^{-t} / t$. Nous pouvons alors écrire

$$
\sum_{n \leqslant x} \nabla_{1}(n) \leqslant x \sum_{\mathrm{e}^{-t} / t<d \leqslant z} \frac{1}{d} \sum_{d<d^{\prime} \leqslant \mathrm{e}^{t} d} \frac{1}{d^{\prime}} \ll x t \sum_{\mathrm{e}^{-t} / t<d \leqslant z} \frac{1}{d} \ll t x \log z .
$$

Il s'ensuit que (3.30) a lieu pour tous les entiers $n \leqslant x$ sauf au plus

$$
\begin{aligned}
\ll \frac{x(\log x) \log z}{3^{w}} & \ll \pi_{w}(x) \frac{(w-1) !(\log x)^{2} \log z}{3^{w}\left(\log _{2} x\right)^{w-1}} \\
& \ll \pi_{w}(x)(\log x)^{Q(\varrho)-\varrho \log 3+1}\left(\log _{2} x\right)^{1 / 2} \log z \ll \frac{\pi_{w}(x)}{(\log x)^{1 / 50}},
\end{aligned}
$$

dès que $\xi \leqslant \frac{1}{110} \log _{2} x$ et la constante $c$ est assez petite, par exemple $c<\frac{1}{30}$. Cela établit bien $(3 \cdot 30)$ pour $j=2$.

La conjonction de $(3 \cdot 30)$ et $(3 \cdot 29)$ fournit donc l'inégalité

$$
V_{t}\left(n_{[w-1]}, 0\right) \leqslant \frac{t 3^{w} w \mathrm{e}^{3 \xi}}{2 \log x}
$$

pour tous les entiers de $\mathcal{E}_{w}(x)$ sauf au plus $\ll \pi_{w}(x) \mathrm{e}^{-\beta \xi}$ exceptions. 
Il reste à examiner l'influence du plus grand facteur premier $p_{w}(n)$ sur la quantité $\nabla^{+}(n, t)$. Nous avons

$$
\nabla^{+}(n, t)=1+V_{t}\left(n_{[w-1]}, 0\right)+2 V_{t}\left(n_{[w-1]}, \log p_{w}(n)\right) \quad\left(n \in \mathcal{E}_{w}^{*}(x)\right) .
$$

Pour majorer le dernier terme, nous considérons la fonction sommatoire

$$
S(x):=\sum_{n \in \mathcal{E}_{w}^{*}(x)} V_{t}\left(n_{[w-1]}, \log p_{w}(n)\right) \leqslant v^{-w} \sum_{n \in \varepsilon_{w}^{*}(x)} v^{\omega(n)} \sum_{\substack{d d^{\prime}|n \\ 0<| \log \left(d^{\prime} / d\right)\left|\leqslant t \\ P^{+}(n)\right| d d^{\prime}}} 1 \quad(0<v \leqslant 1) .
$$

D'après (3.10) avec $k=w$, nous avons $\log P^{+}(n)>\mathrm{e}^{-\xi / \varrho} \log x$ pour tous les entiers de $\mathcal{E}_{w}^{*}(x)$. Compte tenu de la condition $t \leqslant \vartheta^{+}=\mathrm{e}^{-2 \xi} \log x$, il s'ensuit que nous pouvons supposer, dans la dernière somme intérieure,

$$
\min \left(d, d^{\prime}\right)>x_{1}:=\exp \left\{\frac{1}{2} \mathrm{e}^{-\xi / \varrho} \log x\right\} .
$$

Il suit

$$
S(x) \ll v^{-w} \sum_{\substack{x_{1}<d<d^{\prime} \\ 0<\log \left(d^{\prime} / d\right) \leqslant t}} v^{\omega\left(d d^{\prime}\right)} \sum_{m \leqslant x / d d^{\prime}} v^{\omega\left(m d d^{\prime}\right)-\omega\left(d d^{\prime}\right)} .
$$

Nous pouvons majorer la somme intérieure en notant que la fonction $m \mapsto v^{\omega(m D)-\omega(D)}$ est multiplicative pour chaque $D \geqslant 1$. En recourant, par exemple, au théorème III.3.5 de [16], nous obtenons la majoration

$$
S(x) \ll v^{-w} x \sum_{\substack{x_{1}<d \leqslant d^{\prime} \\ d d^{\prime} \leqslant x \\ 0<\log \left(d^{\prime} / d\right) \leqslant t}} \frac{v^{\omega\left(d d^{\prime}\right)}}{\varphi\left(d d^{\prime}\right)}\left(\log \frac{2 x}{d d^{\prime}}\right)^{v-1},
$$

où $\varphi$ désigne la fonction indicatrice d'Euler. En employant alors le théorème de Shiu [11] comme dans [4] — formule (5.28) —, nous obtenons, pour chaque $d$, par sommation d'Abel,

$$
\sum_{\substack{x_{1}<d<d^{\prime} \leqslant x / d \\ 0<\log \left(d^{\prime} / d\right) \leqslant t}} \frac{v^{\omega\left(d d^{\prime}\right)-\omega(d)}}{\varphi\left(d^{\prime}\right)}\left(\log \frac{2 x}{d d^{\prime}}\right)^{v-1} \ll \frac{t d}{v \varphi(d)}\left(\log x_{1}\right)^{v-1}\left(\log \frac{2 x}{d^{2}}\right)^{v-1} .
$$

Nous avons donc finalement établi que

$$
S(x) \ll t x v^{-w-1}\left(\log x_{1}\right)^{v-1} \sum_{x_{1}<d \leqslant \sqrt{x}} \frac{v^{\omega(d)} d}{\varphi(d)^{2}}\left(\log \frac{2 x}{d^{2}}\right)^{v-1} \ll t x v^{-w-2} \mathrm{e}^{(1-v) \xi / \varrho}(\log x)^{3 v-2} .
$$

Pour le choix $v:=\varrho / 3$, il vient

$$
S(x) \ll \frac{t x \mathrm{e}^{3 \xi / 4} 3^{w}}{(\log x)^{Q(\varrho)+1}} \ll \pi_{w}(x) \frac{t 3^{w} \sqrt{w} \mathrm{e}^{3 \xi / 4}}{\log x} .
$$

Il s'ensuit que la majoration

$$
V_{t}\left(n_{[w-1]}, \log p_{w}(n)\right) \leqslant \frac{t 3^{w} w \mathrm{e}^{3 \xi}}{4 \log x}
$$

a lieu pour tous les entiers $n$ de $\varepsilon_{w}(x)$ sauf au plus $\ll \pi_{w}(x) \mathrm{e}^{-\beta \xi}$ exceptions.

En reportant dans (3.32) et en tenant compte de (3.31), nous obtenons bien le résultat annoncé. 


\section{Preuve du Théorème 1.3}

\subsection{Notations}

Le paramètre $\psi$ étant fixé en fonction de $x$ comme indiqué dans l'énoncé, nous nous donnons une constante $c_{0}>0$, qui sera choisie ultérieurement suffisamment petite pour que $2 c_{0} \log \psi \leqslant \frac{1}{2} \log _{2} x$, et nous posons

$$
\begin{aligned}
L & :=\log _{2} x-2 c_{0} \log \psi, \quad M:=\log _{2} x-c_{0} \log \psi, \quad h:=\left[\sqrt{c_{0} \log \psi}\right], \\
k_{j} & :=L+j h \quad(0 \leqslant j \leqslant h), \quad \mathcal{K}:=\left\{k_{j}: 1 \leqslant j \leqslant h\right\} .
\end{aligned}
$$

Nous rappelons la définition de la fonction $Q$ en (1.6).

Enfin, pour tous $n \in \mathbb{N}^{*}, k \in \mathbb{N}$, nous posons

$$
n_{k}:=\prod_{\substack{p \mid n \\ p \leqslant \exp \exp k}} p
$$

Observons que la fonction $n \mapsto n_{k}$ est un analogue multiplicatif de la fonction $n \mapsto n_{[k]}$ considérée dans la preuve du Théorème 1.2.

\subsection{Propriétés arithmétiques des entiers ayant $w$ facteurs premiers}

Nous nous proposons dans ce paragraphe d'établir les propriétés essentielles nécessaires à la preuve du Théorème 1.3 et qui concernent la répartition des facteurs premiers des entiers de $\mathcal{E}_{w}(x)$.

Lemme 4.1. Soit $K \leqslant \log _{2} x$. L'encadrement

$$
\frac{1}{2} \leqslant \frac{\omega(n)-\omega\left(n_{k}\right)}{\left(\log _{2} x\right)-k} \leqslant 2 \quad\left(0 \leqslant k \leqslant \log _{2} x-K\right)
$$

a lieu pour tous les entiers $n \leqslant x$ sauf pour au plus $\ll x \mathrm{e}^{-K / 12}$ exceptions.

Démonstration. Cela résulte immédiatement du lemme 4.5 de [8] en choisissant, dans l'énoncé de ce résultat, $u:=k, v:=\log _{2} x, \xi_{1}=\xi_{2}:=\frac{1}{2} \sqrt{v-u}$ et en sommant sur $k$.

Lemme 4.2. Soit $A>0$. Sous les conditions

$$
\begin{aligned}
& x>16, \quad w \in\left[1, A \log _{2} x\right] \cap \mathbb{N}, \quad \varrho_{0}:=\frac{w}{\log _{2} x}, \\
& 0<\alpha<1, \quad \beta:=\varrho_{0} Q(\alpha), \quad \frac{14}{\beta}+\frac{2}{\beta} \log \left(\frac{1}{1-\mathrm{e}^{-\beta}}\right) \leqslant T \leqslant h,
\end{aligned}
$$

l'inégalité

$$
\left|\left\{k \in \mathcal{K}: \min _{T \leqslant s<h} \frac{\omega\left(n_{k}\right)-\omega\left(n_{k-s}\right)}{s}>\alpha \varrho_{0}\right\}\right| \geqslant \frac{9}{10}|\mathcal{K}|=\frac{9}{10} h
$$

a lieu pour tous les entiers $n$ de $\mathcal{E}_{w}(x)$ sauf au plus $\ll \pi_{w}(x) \mathrm{e}^{-\beta T h / 20}$ exceptions.

Démonstration. Notons $D(n)$ le nombre des indices $k \in \mathcal{K}$ qui ne sont pas comptés dans le membre de gauche de (4·3), de sorte que (4.3) équivaut à $D(n) \leqslant \frac{1}{10} h$. Soit $\ell \in[1, h]$. Nous avons

$$
\left|\left\{n \in \mathcal{E}_{w}(x): D(n) \geqslant \ell\right\}\right| \leqslant \sum_{n \in \mathcal{E}_{w}(x)} \sum_{\substack{\mathcal{H} \subset \mathcal{K} \\|\mathcal{H}|=\ell}} \prod_{k \in \mathcal{H}} \sum_{T \leqslant s<h} \alpha^{\omega\left(n_{k}\right)-\omega\left(n_{k-s}\right)-\alpha \varrho_{0} s} .
$$


En développant le dernier produit et intervertissant les sommations, nous obtenons

$$
\left|\left\{n \in \mathcal{E}_{w}(x): D(n) \geqslant \ell\right\}\right| \leqslant \sum_{\substack{\mathcal{H} \subset \mathcal{K} \\|\mathcal{H}|=\ell}} \sum_{\sigma \in\left[T, h\left[^{\mathcal{H}}\right.\right.} \alpha^{-\alpha \varrho_{0} \operatorname{Tr}(\sigma)} \sum_{n \in \mathcal{E}_{w}(x)} f(n ; \sigma)
$$

où nous convenons que $\left[T, h\left[:=\{s \in \mathbb{N}: T \leqslant s<h\}\right.\right.$ et avons posé $\operatorname{Tr}(\sigma):=\sum_{k \in \mathcal{H}} \sigma(k)$,

$$
f(n ; \sigma):=\prod_{k \in \mathcal{H}} \alpha^{\omega\left(n_{k}\right)-\omega\left(n_{k-\sigma(k)}\right)} \quad\left(\sigma \in \left[T, h\left[^{\mathcal{H}}\right) .\right.\right.
$$

La dernière somme relève du Lemme 3.1. Comme les intervalles $] k-h, k]$, et donc aussi les intervalles $] k-\sigma(k), k]$, sont deux à deux disjoints lorsque $k$ parcourt $\mathcal{H}$, il vient

$$
\sum_{n \in \mathcal{E}_{w}(x)} f(n ; \sigma) \ll_{A} \pi_{w}(x) \exp \left\{\varrho(\alpha-1) \sum_{k \in \mathcal{H}} S_{k}(\sigma)\right\}
$$

où l'on a posé

$$
\varrho=\frac{w-1}{\log _{2} x}, \quad S_{k}(\sigma):=\sum_{k-\sigma(k)<\log _{2} p \leqslant k} \frac{1}{p}=\sigma(k)+O\left(\mathrm{e}^{-L}\right) .
$$

Comme $\ell \ll L$, nous obtenons

$$
\left|\left\{n \in \mathcal{E}_{w}(x): D(n) \geqslant \ell\right\}\right| \ll \pi_{w}(x) \sum_{\substack{\mathcal{H} \subset \mathcal{K} \\|\mathcal{H}|=\ell}} \sum_{\sigma \in[T, h]^{\mathcal{H}}} \mathrm{e}^{\left\{\varrho(\alpha-1)-\alpha \varrho_{0} \log \alpha\right\} \operatorname{Tr}(\sigma)} .
$$

Observant que

$$
\varrho(\alpha-1)-\alpha \varrho_{0} \log \alpha=-\varrho_{0} Q(\alpha)+O\left(1 / \log _{2} x\right)=-\beta+O\left(1 / \log _{2} x\right),
$$

et que $h \ell \ll \log _{2} x$, nous déduisons de (4.4) l'estimation

$$
\begin{aligned}
\left|\left\{n \in \mathcal{E}_{w}(x): D(n) \geqslant \ell\right\}\right| & \ll \pi_{w}(x) \sum_{\substack{\mathcal{H} \subset \mathcal{K} \\
|\mathcal{H}|=\ell}}\left\{\sum_{T \leqslant s<h} \mathrm{e}^{-\beta s}\right\}^{\ell} \\
& \ll \pi_{w}(x)\left(\begin{array}{c}
h \\
\ell
\end{array}\right) \mathrm{e}^{-\beta T \ell}\left(1-\mathrm{e}^{-\beta}\right)^{-\ell} \ll \pi_{w}(x) 2^{h} \mathrm{e}^{-\beta T \ell}\left(1-\mathrm{e}^{-\beta}\right)^{-\ell} .
\end{aligned}
$$

L'hypothèse (4.2) implique $\mathrm{e}^{-\beta T}\left(1-\mathrm{e}^{-\beta}\right)^{-1} \leqslant 2^{-10} \mathrm{e}^{-\beta T / 2}$. Pour le choix $\ell:=1+[h / 10]$, la dernière majoration est donc $\ll \pi_{w}(x) \mathrm{e}^{-\beta T h / 20}$.

Lemme 4.3. Soit $R>0$. Pour $x>16, w \in\left[1, R \log _{2} x\right] \cap \mathbb{N}, 1 \leqslant k \leqslant \log _{2} x, T>0$, on a

$$
\prod_{\substack{p^{\nu} \| n \\ p \leqslant \exp \exp k}} p^{\nu} \leqslant \exp \left(T e^{k}\right)
$$

pour tous les entiers $n$ de $\mathcal{E}_{w}(x)$ sauf pour au plus $\ll_{R} \mathrm{e}^{-T / 2} \pi_{w}(x)$ exceptions. 
Démonstration. Désignons par $n(k)$ le membre de gauche de (4.5) et posons $\alpha:=\frac{1}{2} \mathrm{e}^{-k}$. Le nombre des entiers exceptionnels au sens de l'énoncé ne dépasse pas

$$
\mathrm{e}^{-T / 2} \sum_{n \in \mathcal{E}_{w}(x)} n(k)^{\alpha} \ll_{R} \mathrm{e}^{-T / 2} \pi_{w}(x),
$$

où la majoration résulte immédiatement de $(3 \cdot 1)$.

Lemme 4.4. Sous les conditions $x>16, w \in\left[1,2 \log _{2} x\right] \cap \mathbb{N}$, l'inégalité

$$
\left|\left\{k \in \mathcal{K}: \log n_{k} \leqslant 40 \mathrm{e}^{k}\right\}\right| \geqslant \frac{9}{10}|\mathcal{K}|=\frac{9}{10} h
$$

a lieu pour tous les entiers $n \in \mathcal{E}_{w}(x)$ sauf au plus $\ll \mathrm{e}^{-h} \pi_{w}(x)$ exceptions.

Démonstration. Notons $F(n ; \mathcal{K}):=\left|\left\{k \in \mathcal{K}: \log n_{k}>40 \mathrm{e}^{k}\right\}\right|$. Ainsi (4.6) équivaut à $F(n ; \mathcal{K}) \leqslant h / 10$. Nous avons

$$
F(n ; \mathcal{K}) \leqslant F_{1}(n)+F_{2}(n)
$$

où $F_{1}(n)$ dénombre les indices $j \in[1, h]$ tels que

$$
\log n_{k_{j-1}}>2 h \mathrm{e}^{k_{j-1}}
$$

et $F_{2}(n)$ ceux pour lesquels

$$
\log \left(n_{k_{j}} / n_{k_{j-1}}\right)>39 \mathrm{e}^{k_{j}} .
$$

Soit $\ell:=1+[h / 20]$. D'après le Lemme 4.3 appliqué avec $R:=2$ et $T:=2 h$, nous avons

$$
\sum_{\substack{n \in \mathcal{E}_{w}(x) \\ F_{1}(n) \geqslant \ell}} 1 \leqslant \frac{1}{\ell} \sum_{n \in \mathcal{E}_{w}(x)} F_{1}(n) \ll \mathrm{e}^{-h} \pi_{w}(x),
$$

puisque $|\mathcal{K}|=h \ll \ell$.

Pour majorer $F_{2}(n)$, nous écrivons, avec la même valeur de $\ell$ et en posant $\alpha_{j}=\mathrm{e}^{-k_{j}}$ $(1 \leqslant j \leqslant h)$,

$$
\sum_{\substack{n \in \mathcal{E}_{w}(x) \\ F_{2}(n) \geqslant \ell}} 1 \leqslant \sum_{\substack{\mathcal{J} \subset\{1, \ldots, h\} \\|\mathcal{J}|=\ell}} \mathrm{e}^{-39 \ell} \sum_{n \in \mathcal{E}_{w}(x)} \prod_{j \in \mathcal{J}}\left(n_{k_{j}} / n_{k_{j-1}}\right)^{\alpha_{j}} .
$$

La somme intérieure relève du Lemme 3.1. Elle est

$$
\ll \pi_{w}(x) \exp \left\{\varrho \sum_{j \in \mathcal{J}} \sum_{k_{j-1}<\log _{2} p \leqslant k_{j}} \frac{p^{\alpha_{j}}-1}{p}\right\} \ll \pi_{w}(x) \mathrm{e}^{(\mathrm{e}-1) \varrho \ell},
$$

où l'on a posé $\varrho:=(w-1) / \log _{2} x<2$. Il suit

$$
\sum_{\substack{n \in \mathcal{E}_{w}(x) \\ F_{2}(n) \geqslant \ell}} 1 \ll 2^{h} \mathrm{e}^{-39 \ell+2(\mathrm{e}-1) \ell} \pi_{w}(x) \ll \mathrm{e}^{-h} \pi_{w}(x) .
$$

Le résultat suivant est établi par exemple dans [4], p. 53, ou au lemme III.4.13 de [16].

Lemme 4.5. Soit $f$ une fonction $2 \pi$-périodique, à variation bornée sur $[0,2 \pi]$ et de valeur moyenne

$$
\bar{f}:=\frac{1}{2 \pi} \int_{0}^{2 \pi} f(x) \mathrm{d} x .
$$

Pour tout triplet $(\vartheta, y, z) \in \mathbb{R}^{3}$ tel que $\vartheta \neq 0,1<y<z$, on a

$$
\sum_{y<p \leqslant z} \frac{f(\vartheta \log p)}{p}=\bar{f} \log \left(\frac{\log z}{\log y}\right)+O\left(\frac{V(f)}{|\vartheta| \log y}+\frac{M(f)+(1+|\vartheta|) V(f)}{\mathrm{e}^{\sqrt{\log y}}}\right)
$$

où l'on a posé $M(f):=\sup _{x}|f(x)|, V(f):=\int_{0}^{2 \pi}|\mathrm{d} f(x)|$. 
Pour tous $n \in \mathbb{N}^{*}, \vartheta>0$, posons

$$
\begin{aligned}
\varrho(n, \vartheta) & :=\prod_{p \mid n}(1+2 \cos (\vartheta \log p)), \\
\omega_{\vartheta}(n) & :=\omega(n, \exp (1 / \vartheta)), \quad \mathcal{R}(n, \vartheta):=\frac{\varrho(n, \vartheta)^{2}}{3^{\omega(n)+\omega_{\vartheta}(n)}} .
\end{aligned}
$$

Le résultat suivant peut être établi en appliquant successivement les Lemmes 3.1 et 4.5. Nous omettons les détails qui sont semblables à ceux de la preuve du lemme 51.3 de [4].

Lemme 4.6. Soit $R>0$. On a, uniformément pour $x>16, w \in\left[1, R \log _{2} x\right] \cap \mathbb{N}$, $\varrho:=w / \log _{2} x, \vartheta>0$ et $k \in \mathbb{N}$,

$$
\sum_{n \in \mathcal{E}_{w}(x)} \mathcal{R}\left(n_{k}, \vartheta\right) \ll_{R}\{\log (3+\vartheta)\}^{4 \varrho} \pi_{w}(x) .
$$

On en déduit immédiatement le résultat suivant.

Lemme 4.7. Soit $R>0$. Sous les conditions $x>16, w \in\left[1, R \log _{2} x\right] \cap \mathbb{N}, \varrho:=w / \log _{2} x$, $V>0, H>1, k \in \mathbb{N}$, l'inégalité

$$
\int_{1}^{H} \mathcal{R}\left(n_{k}, \vartheta\right) \mathrm{d} \vartheta \leqslant V H\{\log (3+H)\}^{4 \varrho}
$$

a lieu pour tous les entiers $n$ de $\mathcal{E}_{w}(x)$ sauf pour au plus $\ll \pi_{w}(x) / V$ exceptions.

Lemme 4.8. Soient $R>0, \delta>1$. Sous les conditions $x>16, w \in\left[1, R \log _{2} x\right] \cap \mathbb{N}$, $V>0$, l'inégalité

$$
\left|\left\{k \in \mathcal{K}: \int_{\mathrm{e}^{h-k}}^{1} \mathcal{R}\left(n_{k}, \vartheta\right) \frac{\mathrm{d} \vartheta}{\vartheta^{\delta}} \leqslant V \mathrm{e}^{(\delta-1)(k-h)}\right\}\right| \geqslant \frac{9}{10} h
$$

a lieu pour tous les entiers $n$ de $\mathcal{E}_{w}(x)$ sauf au plus $\ll_{R, \delta} \pi_{w}(x) / V$ exceptions.

Démonstration. Posons $\ell:=1+[h / 10]$, et désignons par $G(n ; \mathcal{K})$ le nombre des indices $k \in \mathcal{K}$ qui ne sont pas comptés dans le membre de gauche de (4.9). Nous avons

$$
\begin{aligned}
\sum_{\substack{n \in \mathcal{E}_{w}(x) \\
G(n ; \mathcal{K}) \geqslant \ell}} 1 & \leqslant \frac{1}{\ell} \sum_{n \in \mathcal{E}_{w}(x)} G(n ; \mathcal{K}) \leqslant \frac{1}{\ell V} \sum_{k \in \mathcal{K}} \mathrm{e}^{(1-\delta)(k-h)} \int_{\mathrm{e}^{h-k}}^{1} \sum_{n \in \mathcal{E}_{w}(x)} \mathcal{R}\left(n_{k}, \vartheta\right) \frac{\mathrm{d} \vartheta}{\vartheta^{\delta}} \\
& \ll R \frac{\pi_{w}(x)}{(\delta-1) V},
\end{aligned}
$$

où la dernière somme en $n$ a été estimée grâce au Lemme 4.6.

Lemme 4.9. Soit $R>0$. Sous les conditions

$x>16, w \in\left[1, R \log _{2} x\right] \cap \mathbb{N}, J \in \mathbb{N}, k_{j} \in \mathbb{N}(0 \leqslant j \leqslant J), 0=k_{0}<k_{1}<\ldots<k_{J}$,

nous avons

$$
\sum_{n \in \mathcal{E}_{w}(x)} \prod_{1 \leqslant j \leqslant J} \mathcal{R}\left(n_{k_{j}}, \vartheta_{j}\right) \leqslant \mathrm{e}^{c_{2} R J} \pi_{w}(x) \quad\left(\mathrm{e}^{-k_{j}} \leqslant \vartheta_{j} \leqslant \mathrm{e}^{-k_{j-1}}, 1 \leqslant j \leqslant J\right)
$$

où $c_{2}$ est une constante absolue positive. 
Démonstration. Pour tous $k \in \mathbb{N}, \vartheta>0$, nous avons

$$
\begin{aligned}
\mathcal{R}\left(n_{k}, \vartheta\right) \leqslant & \prod_{\substack{p \mid n \\
1 / \vartheta<\log p \leqslant \mathrm{e}^{k}}} \frac{1}{3}(1+2 \cos (\vartheta \log p))^{2} \\
= & \prod_{\substack{p \mid n \\
1 / \vartheta<\log p \leqslant \mathrm{e}^{k}}}\left(1+\frac{2}{3} \cos (2 \vartheta \log p)+\frac{4}{3} \cos (\vartheta \log p)\right) .
\end{aligned}
$$

Par hypothèse, les intervalles $\left.] 1 / \vartheta_{j}, \mathrm{e}^{k_{j}}\right](1 \leqslant j \leqslant J)$ sont deux à deux disjoints. Il suit

$$
\prod_{1 \leqslant j \leqslant J} \mathcal{R}\left(n_{k_{j}}, \vartheta_{j}\right) \leqslant f(n)
$$

où $f$ est la fonction fortement multiplicative définie par

$$
f(p):=1+\frac{2}{3} \sum_{1 \leqslant j \leqslant J} \mathbf{1}_{\left[1 / \vartheta_{j}, \mathrm{e}^{k_{j}}\right]}(\log p)\left\{\cos \left(2 \vartheta_{j} \log p\right)+2 \cos \left(\vartheta_{j} \log p\right)\right\}
$$

D'après le Lemme 3.1, nous pouvons écrire, avec $\varrho:=(w-1) / \log _{2} x$

$$
\sum_{n \in \mathcal{E}_{w}(x)} f(n) \ll \pi_{w}(x) \exp \left\{\frac{2}{3} \varrho \sum_{1 \leqslant j \leqslant J} \sum_{\exp \left(1 / \vartheta_{j}\right)<p \leqslant \exp ^{k_{j}}} \frac{\cos \left(2 \vartheta_{j} \log p\right)+2 \cos \left(\vartheta_{j} \log p\right)}{p}\right\} .
$$

Les sommes intérieures étant uniformément bornées en vertu du Lemme 4.5, nous obtenons bien la majoration requise $(4 \cdot 10)$.

Lemme 4.10. Soit $R>0$. Il existe une constante $c_{3}=c_{3}(R)>0$ telle que, pour tout $\delta>1$, et uniformément sous les conditions $x>16, w \in\left[1, R \log _{2} x\right] \cap \mathbb{N}$, l'inégalité

$$
\left|\left\{k \in \mathcal{K}: \quad \int_{\mathrm{e}^{-k}}^{\mathrm{e}^{h-k}} \mathcal{R}\left(n_{k}, \vartheta\right) \frac{\mathrm{d} \vartheta}{\vartheta^{\delta}} \leqslant \frac{c_{3} \mathrm{e}^{(\delta-1) k}}{\delta-1}\right\}\right| \geqslant \frac{9}{10} h
$$

ait lieu pour tous les entiers $n$ de $\mathcal{E}_{w}(x)$ sauf au plus $\ll_{R, \delta} \mathrm{e}^{-h} \pi_{w}(x)$ exceptions.

Démonstration. Posons $\ell:=1+[h / 10]$, et désignons par $B(n ; \mathcal{K})$ le nombre des indices $k \in \mathcal{K}$ qui ne sont pas comptés dans le membre de gauche de (4·11). Nous avons

$$
\begin{aligned}
& \sum_{\substack{n \in \mathcal{E}_{w}(x) \\
B(n ; \mathcal{K}) \geqslant \ell}} 1 \leqslant \sum_{n \in \mathcal{E}_{w}(x)}\left(\frac{\delta-1}{c_{3}}\right)^{\ell} \sum_{\substack{\mathcal{J} \subset\{1, \ldots, h\} \\
|\mathcal{I}|=\ell}} \prod_{j \in \mathcal{J}} \mathrm{e}^{(1-\delta) k_{j}} \int_{\mathrm{e}^{-k_{j}}}^{\mathrm{e}^{-k_{j}-1}} \mathcal{R}\left(n_{k_{j}}, \vartheta\right) \frac{\mathrm{d} \vartheta}{\vartheta^{\delta}} \\
& =\left(\frac{\delta-1}{c_{3}}\right)^{\ell} \sum_{\substack{\mathcal{J} \subset\{1, \ldots, h\} \\
|\mathcal{J}|=\ell}}\left\{\prod_{j \in \mathcal{J}} \mathrm{e}^{(1-\delta) k_{j}}\right\} \int_{\Delta(\mathcal{J})} \sum_{n \in \mathcal{E}_{w}(x)} \prod_{j \in \mathcal{J}} \mathcal{R}\left(n_{k_{j}}, \vartheta_{j}\right) \prod_{i \in \mathcal{J}} \frac{\mathrm{d} \vartheta_{j}}{\vartheta_{j}^{\delta}}
\end{aligned}
$$

où l'on a posé $\Delta(\mathcal{J}):=\prod_{j \in \mathcal{J}}\left[\mathrm{e}^{-k_{j}}, \mathrm{e}^{-k_{j-1}}\right]$. D'après le Lemme 4.9, la somme intérieure est $\ll \mathrm{e}^{c_{2} R \ell} \pi_{w}(x)$. Il s'ensuit que

$$
\sum_{\substack{n \in \mathcal{E}_{w}(x) \\ B(n ; \mathcal{K}) \geqslant \ell}} 1 \ll \pi_{w}(x) 2^{h} c_{3}^{-\ell} \mathrm{e}^{c_{2} R \ell}
$$

Comme $h \leqslant 10 \ell$, le choix $c_{3}:=2^{20} \mathrm{e}^{c_{2} R+10}$ permet de conclure. 


\subsection{Préparation}

Dans ce paragraphe, nous mettons en place le cadre final de la démonstration du Théorème 1.3.

Dans toute la suite, nous posons

$$
\left.\delta:=\frac{1}{2}\{1+\log 3\} \approx 1,04931, \quad \alpha:=\delta / \log 3 \in\right] 0,1[.
$$

Étant donnés $B>0$ et $x>16$, nous considérons des paramètres $\psi, w$ tels que

$$
\left(\log _{2} x\right)^{10} \leqslant \psi \leqslant(\log x)^{B}, \quad w \in \mathbb{N}, \quad\left|w-\log _{2} x\right| \leqslant(\log \psi)^{1 / 4}\left(\log _{2} x\right)^{1 / 2},
$$

et nous posons

$$
\varrho_{0}=\varrho_{0}(w, x):=\frac{w}{\log _{2} x}, \quad \Theta^{-}=\Theta^{-}(w, x, \psi)=\frac{\log x}{3^{w}} \sqrt{\psi}, \quad \Theta^{+}=\Theta^{+}(x, \psi):=\frac{\log x}{\sqrt{\psi}} .
$$

et rappelons les notations $(4 \cdot 1)$.

Pour tous $c>0, T>0$, nous désignons par $\mathcal{S}_{w}(x ; c, T)$ l'ensemble des entiers $n$ de $\mathcal{E}_{w}(x)$ qui vérifient les six propriétés suivantes :
(i) $\frac{1}{2} \leqslant \frac{w-\omega\left(n_{k}\right)}{\log _{2} x-k} \leqslant 2 \quad(k \in \mathcal{K}) ;$
(ii) $\left|\left\{k \in \mathcal{K}: \min _{T \leqslant s \leqslant k} \frac{\omega\left(n_{k}\right)-\omega\left(n_{k-s}\right)}{s}>\alpha\right\}\right| \geqslant \frac{9}{10} h$;
(iii) $\left|\left\{k \in \mathcal{K}: \log n_{k} \leqslant 40 e^{k}\right\}\right| \geqslant \frac{9}{10} h$;
(iv) $\left|\left\{k \in \mathcal{K}: \int_{\mathrm{e}^{-k}}^{1} \mathcal{R}\left(n_{k}, \vartheta\right) \mathrm{d} \vartheta / \vartheta^{\delta} \leqslant c \mathrm{e}^{(\delta-1) k}\right\}\right| \geqslant \frac{4}{5} h$;
(v) $\int_{1}^{1 / \Theta^{-}} \mathcal{R}\left(n_{k}, \vartheta\right) \mathrm{d} \vartheta \leqslant \mathrm{e}^{h}\left(\log _{2} x\right)^{4 \varrho_{0}} / \Theta^{-} \quad(k \in \mathcal{K}) ;$
(vi) $\prod_{\log _{2} p \leqslant M} p^{\nu} \| n, p^{\nu} \leqslant x^{1 / 10}$, où $M$ est défini en $(4 \cdot 1)$.

Le résultat suivant découle immédiatement des Lemmes 4.1, 4.2, 4.4, 4.8, 4.10, 4.7 et 4.3.

Lemme 4.11. Il existe deux constantes $T>0, c>0$ telles que, pour toute constante $B>0$, tout nombre réel $x$ suffisamment grand, et sous les conditions (4.13), on ait

$$
\left|\mathcal{S}_{w}(x ; c, T)\right| \geqslant\left\{1-\mathrm{e}^{-(\delta-1) h}\right\} \pi_{w}(x) .
$$

Pour chaque $n \in \mathcal{S}_{w}(x ; c, T)$, notons

$$
k_{1}(n)<k_{2}(n)<\ldots<k_{v}(n)
$$

les $v:=[h / 2]$ plus petits entiers $k$ distincts qui sont simultanément comptés dans les ensembles apparaissant en (ii), (iii), (iv), et désignons par $\mathcal{K}_{n}$ leur ensemble.

Compte tenu de (4·1), nous déduisons de (i) que, pour tout $n \in \mathcal{S}_{w}(x ; c, T)$, nous avons

$$
\frac{1}{2} c_{0} \log \psi \leqslant w-\omega\left(n_{k}\right) \leqslant 4 c_{0} \log \psi \quad\left(k \in \mathcal{K}_{n}\right) .
$$

Posons, avec la notation $(3 \cdot 19)$,

$$
\mathcal{L}_{t}(m):=\left\{z \in \mathbb{R}: V_{t}(m, z)>t / \Theta^{-}\right\}, \quad \lambda_{t}(m):=\operatorname{mes} \mathcal{L}_{t}(m) .
$$


Lemme 4.12 (Lemme fondamental). Sous les conditions (4.13) et uniformément pour $x$ assez grand, $n \in \mathcal{S}_{w}(x ; c, T), k \in \mathcal{K}_{n}$ et $\Theta^{-} \leqslant t \leqslant \Theta^{+}$, nous avons

$$
\lambda_{t}\left(n_{k}\right) \gg \mathrm{e}^{k} .
$$

Démonstration. Nous employons la méthode développée dans [4] pour établir l'inégalité (5.49), p. 109. Les détails étant très voisins, nous nous limitons à des indications succinctes.

Pour tout entier $m$ sans facteur carré, nous avons

$$
3^{\omega(m)} \leqslant \frac{t+\log m}{\Theta^{-}}+\left\{2 \pi \lambda_{t}(m) \int_{-1 / \Theta^{-}}^{1 / \Theta^{-}} \varrho(m, \vartheta)^{2} \mathrm{~d} \vartheta\right\}^{1 / 2}
$$

Appliquons cette inégalité pour $t \in\left[\Theta^{-}, \Theta^{+}\right]$et $m=n_{k}$ lorsque $n \in \mathcal{S}_{w}(x ; c, T), k \in \mathcal{K}_{n}$, en tenant compte du fait que $(4 \cdot 1),(4 \cdot 15)$ et (iii) impliquent, si la constante $c_{0}$ est choisie assez petite,

$$
\frac{\log n_{k}}{\Theta^{-}} \leqslant \frac{40 \mathrm{e}^{k} 3^{w}}{\sqrt{\psi} \log x} \leqslant \frac{1}{4} 3^{\omega\left(n_{k}\right)} \quad \text { et } \quad \frac{t}{\Theta^{-}} \leqslant \frac{\Theta^{+}}{\Theta^{-}}=\frac{3^{w}}{\psi} \leqslant \frac{1}{4} 3^{\omega\left(n_{k}\right)}
$$

Posant

$$
I(m):=\int_{0}^{1 / \Theta^{-}} \frac{\varrho(m, \vartheta)^{2}}{3^{2 \omega(m)}} \mathrm{d} \vartheta \quad(m \geqslant 1)
$$

il résulte de ce qui précède que

$$
\lambda_{t}\left(n_{k}\right) I\left(n_{k}\right) \geqslant \frac{1}{16 \pi} \quad\left(n \in \mathcal{S}_{w}(x ; c, T), k \in \mathcal{K}_{n}\right),
$$

de sorte que la preuve du Lemme 4.12 se réduit à établir que

$$
I\left(n_{k}\right) \ll \mathrm{e}^{-k} .
$$

À cette fin, nous introduisons les contributions à l'intégrale $I\left(n_{k}\right)$ des domaines d'intégration $\left[0, \mathrm{e}^{T-k}\right],\left[\mathrm{e}^{T-k}, 1\right]$ et $\left[1, \max \left(1,1 / \Theta^{-}\right)\right]$. Nous notons ces contributions respectives $I_{j}\left(n_{k}\right)(1 \leqslant j \leqslant 3)$. Ici et dans la suite, le paramètre $T$ est tel que défini au Lemme 4.11.

Nous avons trivialement $I_{1}\left(n_{k}\right) \leqslant \mathrm{e}^{T-k}$. De plus, les propriétés (ii) et (iv) permettent d'écrire

$$
I_{2}\left(n_{k}\right):=\int_{\mathrm{e}^{T-k}}^{1} \mathcal{R}\left(n_{k}, \vartheta\right) 3^{-\left(\omega\left(n_{k}\right)-\omega_{\vartheta}\left(n_{k}\right)\right)} \mathrm{d} \vartheta \leqslant 3 \int_{\mathrm{e}^{-k}}^{1} \mathcal{R}\left(n_{k}, \vartheta\right) 3^{-\alpha \log \left(\vartheta \mathrm{e}^{k}\right)} \mathrm{d} \vartheta \ll \mathrm{e}^{-k} .
$$

Enfin, dans le cas non trivial où $\Theta^{-}<1$, nous avons, d'après $(4 \cdot 13),(4 \cdot 15)$ et $(\mathrm{v})$,

$$
\begin{aligned}
I_{3}\left(n_{k}\right) & \leqslant 3 \int_{1}^{1 / \Theta^{-}} \mathcal{R}\left(n_{k}, \vartheta\right) 3^{-\omega\left(n_{k}\right)} \mathrm{d} \vartheta \\
& \ll \frac{\psi^{4 c_{0}}\left(\log _{2} x\right)^{4 \varrho} \mathrm{e}^{h}}{3^{w} \Theta^{-}} \ll \psi^{3 c_{0}-1 / 2}\left(\log _{2} x\right)^{4 \varrho_{0}} \mathrm{e}^{-k} \ll \mathrm{e}^{-k}
\end{aligned}
$$

sous réserve de diminuer convenablement la valeur de la constante $c_{0}$.

\subsection{Complétion de l'argument}

Nous utilisons la technique développée dans la preuve de la proposition 1 de [9] ou du théorème 1 de [12] et reposant sur l'argument mis en place dans [6]. 
Soient $B, x, w, \psi, t$ satisfaisant (1.5). Observons d'abord, qu'il suffit d'établir que l'inégalité

$$
\nabla(n, t) \geqslant t / \Theta^{-} \quad\left(\Theta^{-} \leqslant t \leqslant \Theta^{+}\right) .
$$

a lieu avec la majoration annoncée pour le nombre d'exceptions. En effet, si l'entier $n$ de $\mathcal{E}_{w}(x)$ vérifie $(4 \cdot 16)$, on a

$$
\nabla(n, t) \geqslant \nabla\left(n, \Theta^{+}\right) \geqslant \frac{\Theta^{+}}{\Theta^{-}}=\frac{3^{w}}{\psi} \geqslant \frac{3^{w} t}{\psi \log x} \quad\left(\Theta^{+}<t \leqslant \log x\right),
$$

alors que la minoration requise découle de l'inégalité triviale $\nabla(n, t) \geqslant 1$ lorsque $t<\Theta^{-}$. Nous pouvons donc nous limiter à établir $(4 \cdot 16)$.

Le nombre $\delta$ étant défini par $(4 \cdot 12)$, les paramètres $c$ et $T$ étant fixés comme indiqué au Lemme 4.11 et la suite $\left\{k_{j}(n)\right\}_{j=1}^{v}$ étant définie, pour chaque entier $n$ de $\mathcal{S}_{w}(x ; c, T)$, comme précisé en $(4 \cdot 14)$, nous posons

$$
\begin{aligned}
& E^{w}:=\left\{n \in \mathcal{E}_{w}(x): \nabla(n, t) \leqslant t / \Theta^{-}\right\}, \\
& \mathcal{T}_{s}^{w}:=\left\{n \in \mathcal{S}_{w}(x ; c, T): \nabla\left(n_{k_{s}(n)}, t\right) \leqslant t / \Theta^{-}\right\} \quad(1 \leqslant s \leqslant v:=[h / 2]) .
\end{aligned}
$$

On a clairement $E^{w} \cap \mathcal{S}_{w}(x ; c, T) \subset \mathcal{T}_{v}^{w}$. En vertu du Lemme 4.11, cela implique que

$$
\left|E^{w}\right| \leqslant\left|\mathcal{T}_{v}^{w}\right|+\pi_{w}(x) \mathrm{e}^{-(1-\delta) h} .
$$

Nous allons estimer $\left|\mathcal{T}_{s}^{w}\right|(1 \leqslant s \leqslant v)$ par récurrence sur l'indice $s$. Pour chaque $s$, nous formons une partition de $\mathcal{T}_{s}$ selon les valeurs de $k_{s}(n)$ : notant

$$
\mathcal{T}_{s, k}^{w}:=\mathcal{T}_{s}^{w} \cap\left\{n \in \mathcal{S}_{w}(x ; c, T): k_{s}(n)=k\right\} \quad(k \in \mathcal{K}),
$$

nous avons

$$
\mathcal{T}_{s}^{w}=\bigcup_{k \in \mathcal{K}} \mathcal{T}_{s, k}^{w} \quad(1 \leqslant s \leqslant v) .
$$

Nous introduisons alors, pour chaque $s$, les ensembles deux à deux disjoints

$$
\mathcal{M}_{s, k}^{w}:=\left\{m \leqslant x: \exists n \in \mathcal{T}_{s, k}^{w}, m=\prod_{\log _{2} p^{\nu} \| n} p^{\nu}\right\}
$$

et nous considérons l'ensemble $\mathcal{D}_{s, k}^{w}$ des entiers $n$ de $\mathcal{E}_{w}(x)$ représentables sous la forme $n=m p q b$ où $m \in \mathcal{M}_{s, k}^{w}, P^{-}(b)>\exp \left(80 \mathrm{e}^{k}\right)$ et $(p, q)$ est un couple de nombres premiers assujetti aux conditions suivantes :

$$
\left\{\begin{array}{l}
\mathrm{e}^{k}<\log p \leqslant 40 \mathrm{e}^{k}, \\
\log p<\log q \leqslant \log p+40 \mathrm{e}^{k}, \\
\log q-\log p \in \mathcal{L}_{t}\left(m_{k}\right) .
\end{array}\right.
$$

Ainsi, les $\mathcal{D}_{s, k}^{w}$ sont également deux à deux disjoints et l'on vérifie sans peine l'inclusion

$$
\bigcup_{k \in \mathcal{K}} \mathcal{D}_{s, k}^{w} \cap \mathcal{S}_{w}(x ; c, T) \subset \mathcal{T}_{s}^{w} \backslash \mathcal{T}_{s+1}^{w} \quad(1 \leqslant s<v) .
$$


Par le Lemme 4.11, nous en déduisons que

$$
\left|\mathcal{T}_{s+1}^{w}\right| \leqslant\left|\mathcal{T}_{s}^{w}\right|-\sum_{k \in \mathcal{K}}\left|\mathcal{D}_{s, k}^{w}\right|+\pi_{w}(x) \mathrm{e}^{-(1-\delta) h} \quad(1 \leqslant s<v) .
$$

Il reste à minorer $\mathcal{D}_{s, k}^{w}$. D'une part, nous avons

$$
\left|\mathcal{D}_{s, k}^{w}\right| \geqslant \sum_{m \in \mathcal{M}_{s, k}^{w}} \sum_{(p, q)} \sum_{b}^{*} 1
$$

où l'astérisque indique que la sommation porte sur les entiers $b \leqslant x_{1}:=x /(m p q)$ tels que $P^{-}(b)>y=\exp \left(\mathrm{e}^{k+5}\right)$ et $\omega(b)=j:=w-\omega(m)-2$. Les hypothèses du Lemme 3.2 sont alors satisfaites et l'on a $j \ll \log u$ avec $u:=\log x_{1} / \log y$. D'où

$$
\sum_{b}^{*} 1 \gg \frac{x_{1}\left(\log _{2} x-k\right)^{j-1}}{\left(\log x_{1}\right)(j-1) !} \gg \frac{x\left(\log _{2} x-k\right)^{j}}{(m p q) j ! \log x}
$$

puisque $j \asymp \log _{2} x-k$. Par ailleurs, le Lemme 4.12 permet, par une manipulation standard reposant sur une application d'une forme forte du théorème des nombres premiers, d'obtenir la minoration

$$
\sum_{p} \frac{1}{p} \sum_{q} \frac{1}{q} \gg 1
$$

Nous pouvons donc énoncer que

$$
\mathcal{D}_{s, k}^{w} \gg \frac{x\left(\log _{2} x-k\right)^{j}}{j ! \log x} \sum_{m \in \mathcal{M}_{s, k}^{w}} \frac{1}{m} .
$$

D'autre part, nous avons

$$
\left|\mathcal{T}_{s, k}^{w}\right| \leqslant \sum_{m \in \mathcal{M}_{s, k}^{w}} \sum_{b_{2}}^{* *} 1
$$

où la double astérisque indique que la somme ainsi mentionnée porte sur les entiers $b_{2} \leqslant x_{2}:=x / m$ tels que $P^{-}\left(b_{2}\right) \geqslant \exp \left(\mathrm{e}^{k}\right)$ et $\omega\left(b_{2}\right)=w-\omega(m)=j+2$. En appliquant le Lemme 3.2 et la propriété (vi), on obtient

$$
\sum_{b_{2}}^{* *} 1 \ll \frac{x_{2}\left(\log _{2} x-k\right)^{j+1}}{(j+1) ! \log x_{2}} \ll \frac{x\left(\log _{2} x-k\right)^{j}}{m j ! \log x} .
$$

Nous déduisons de $(4 \cdot 20),(4 \cdot 21)$ et $(4 \cdot 22)$ l'existence d'une constante $c_{5}>0$ telle que

$$
\left|\mathcal{D}_{s, k}^{w}\right| \geqslant c_{5}\left|\mathcal{T}_{s, k}^{w}\right| \text {. }
$$

Supposons dans un premier temps que $\left|E^{w}\right| \geqslant\left(1+2 / c_{5}\right) \mathrm{e}^{-(1-\delta) h} \pi_{w}(x)$. Compte tenu de $(4 \cdot 17)$, pour tout entier $s \in[1, v]$, nous avons

$$
\left|\mathcal{T}_{s}^{w}\right| \geqslant\left|\mathcal{T}_{v}^{w}\right| \geqslant\left|E^{w}\right|-\pi_{w}(x) \mathrm{e}^{-(1-\delta) h} \geqslant\left(2 / c_{5}\right) \pi_{w}(x) \mathrm{e}^{-(1-\delta) h} .
$$

Il découle donc des relations $(4 \cdot 23),(4 \cdot 19)$ et $(4 \cdot 18)$ que

$$
\left|\mathcal{T}_{s+1}^{w}\right| \leqslant\left(1-\frac{1}{2} c_{5}\right)\left|\mathcal{T}_{s}^{w}\right| \quad(1 \leqslant s<v)
$$

ce qui implique par récurrence sur $s$

$$
\left|\mathcal{T}_{v}^{w}\right| \leqslant\left(1-\frac{1}{2} c_{5}\right)^{v-1}\left|\mathcal{T}_{1}^{w}\right| \leqslant \pi_{w}(x) \mathrm{e}^{-c_{6} h} .
$$

Compte tenu de $(4 \cdot 17)$, nous avons donc finalement établi que l'on a en toute circonstance

$$
\left|E^{w}\right| \ll\left\{\mathrm{e}^{-c_{6} h}+\mathrm{e}^{-(1-\delta) h}\right\} \pi_{w}(x) \ll \mathrm{e}^{-c_{7} h} \pi_{w}(x) \ll \pi_{w}(x) \mathrm{e}^{-c_{2} \sqrt{\log \psi}} .
$$




\section{Preuve du Corollaire 1.6}

Pour $n$ assez grand et tel que, par exemple, $3^{\omega(n)}>(\log n)^{21 / 20}$, nous posons

$$
G(n):=3^{\omega(n)} / \log n,
$$

et $L_{n}:=\exp \left\{\left(A+\varepsilon_{n}\right)\left(\log _{2} n\right)^{2}\right\}$ où $A$ est la constante apparaissant dans (1.10) et $\varepsilon_{n}$ est choisi dans $[0,1]$ de façon que

$$
J_{n}:=\frac{\log \left\{(\log n) G(n) L_{n}\right\}}{2 \log L_{n}}=\frac{\log \left\{3^{\omega(n)} L_{n}\right\}}{2 \log L_{n}} \in \mathbb{N} .
$$

Définissons alors la suite

$$
T_{j}:=G(n)^{-1} L_{n}^{2 j-1} \quad(j \geqslant 0),
$$

qui vérifie donc $T_{J_{n}}=\log n$. D'après le Corollaire 1.4, nous avons

$$
T_{0}<E(n) \leqslant T_{1} \quad \text { pp }
$$

alors que $(1 \cdot 10)$ permet d'écrire

$$
L_{n}^{2 j-2} \leqslant \nabla^{+}\left(n, T_{j+1}\right)-\nabla^{+}\left(n, T_{j}\right) \leqslant L_{n}^{2 j+3} \quad\left(0 \leqslant j<J_{n}\right) \quad \text { pp },
$$

où nous avons tenu compte de $(5 \cdot 1)$ pour le cas $j=0$. Par ailleurs, $f$ étant nécessairement continue, il existe une suite $\left\{\xi_{j}\right\}_{j=0}^{J_{n}-1}$ telle que

$$
T_{j} \leqslant \xi_{j} \leqslant T_{j+1} \quad\left(0 \leqslant j<J_{n}\right), \quad \int_{T_{0}}^{\log n} f(t) \mathrm{d} t=\sum_{0 \leqslant j<J_{n}} f\left(\xi_{j}\right)\left\{T_{j+1}-T_{j}\right\} .
$$

Il suit, pour presque tout entier $n$,

$$
\begin{aligned}
\vartheta(n ; f) & \leqslant L_{n}^{2 K(f)} \sum_{0 \leqslant j<J_{n}} f\left(\xi_{j}\right)\left\{\nabla^{+}\left(n, T_{j+1}\right)-\nabla^{+}\left(n, T_{j}\right)\right\} \leqslant \sum_{0 \leqslant j<J_{n}} f\left(\xi_{j}\right) L_{n}^{2 j+3+2 K(f)} \\
& \leqslant 2 G(n) L_{n}^{2+2 K(f)} \sum_{0 \leqslant j<J_{n}} f\left(\xi_{j}\right)\left\{T_{j+1}-T_{j}\right\}=2 G(n) L_{n}^{2+2 K(f)} \int_{T_{0}}^{\log n} f(t) \mathrm{d} t .
\end{aligned}
$$

Comme

$$
\int_{T_{0}}^{1 / G(n)} f(t) \mathrm{d} t \leqslant L_{n}^{K(f)} \int_{1 / G(n)}^{L_{n} / G(n)} f(t) \mathrm{d} t \leqslant L_{n}^{K(f)} \int_{1 / G(n)}^{\log n} f(t) \mathrm{d} t,
$$

nous avons établi la majoration contenue dans (1·11).

Pour montrer la minoration, nous écrivons, compte tenu de $(5 \cdot 1)$ et $(5 \cdot 2)$,

$$
\begin{aligned}
\vartheta(n ; f) & \geqslant \frac{1}{2 L_{n}^{2 K(f)}} \sum_{0 \leqslant j<J_{n}} f\left(\xi_{j}\right)\left\{\nabla^{+}\left(n, T_{j+1}\right)-\nabla^{+}\left(n, T_{j}\right)\right\} \geqslant \frac{1}{2} \sum_{0 \leqslant j<J_{n}} f\left(\xi_{j}\right) L_{n}^{2 j-2-2 K(f)} \\
& \geqslant \frac{G(n)}{4 L_{n}^{3+2 K(f)}} \sum_{0 \leqslant j<J_{n}} f\left(\xi_{j}\right)\left\{T_{j+1}-T_{j}\right\}=\frac{G(n)}{4 L_{n}^{3+2 K(f)}} \int_{T_{0}}^{\log n} f(t) \mathrm{d} t \quad \mathrm{pp} .
\end{aligned}
$$

Cela complète la démonstration.

Remerciements. Les auteurs tiennent à exprimer leurs plus chaleureux remerciements à l'arbitre, dont la lecture exhaustive et méticuleuse a permis de rectifier un certain nombre d'imprécisions dans la version initiale de ce travail. 


\section{Bibliographie}

[1] K. Alladi, The distribution of $\nu(n)$ in the sieve of Eratosthenes, Quart. J. Math. (2), 33 (1982), $129-148$.

[2] M. Balazard, Unimodalité de la distribution du nombre de diviseurs premiers d'un entier, Ann. Inst. Fourier, Grenoble 40, 2 (1990), 255-270.

[3] P. Erdős, On the density of some sequences of integers, Bull. Amer. Math. Soc. 54 (1948), 685-692.

[4] R.R. Hall \& G. Tenenbaum, Divisors, Cambridge University Press (1988).

[5] M. Mendès France \& G. Tenenbaum, Systèmes de points, diviseurs, et structure fractale, Bull. Soc. Math. de France 121 (1993), 197-225.

[6] H. Maier \& G. Tenenbaum, On the set of divisors of an integer, Invent. Math. 76 (1984), 121-128.

[7] H. Maier \& G. Tenenbaum, On the normal concentration of divisors, J. London Math. Soc. (2) 31 (1985), 393-400.

[8] H. Maier \& G. Tenenbaum, On the normal concentration of divisors, 2, Math. Proc. Camb. Phil. Soc., à paraître.

[9] A. Raouj, Sur la densité de certains ensembles de multiples, 1, Acta Arith. 69 (1995),121-152.

[10] A. Raouj \& G.Tenenbaum, Sur l'écart quadratique moyen des diviseurs d'un entier normal, Math. Proc. Camb. Phil. Soc. 126 (1999), 399-415.

[11] P. Shiu, A Brun-Titchmarsh theorem for multiplicative functions, J. reine angew. Math. 313 (1980), 161-170.

[12] A. Stef, L'ensemble exceptionnel dans la conjecture d'Erdös concernant la proximité des diviseurs, Thèse de l'Université de Nancy I, 1992.

[13] G. Tenenbaum, Sur la concentration moyenne des diviseurs, Comment. Math. Helvetici 60 (1985), 411-428.

[14] G. Tenenbaum, A rate estimate in Billingsley's theorem for the size distribution of large prime factors, Quart. J. Math. (Oxford) 51 (2000), 385-403.

[15] G. Tenenbaum, Sur l'écart quadratique moyen des diviseurs d'un entier normal, 2, Math. Proc. Camb. Phil. Soc. 138 (2005), 1-8.

[16] G. Tenenbaum, Introduction à la théorie analytique et probabiliste des nombres, troisième édition, coll. Échelles, Belin, 2008, 592 pp.

\author{
Abdelaziz Raouj \\ Département de mathématiques \\ Université Caddi Ayyad \\ Faculté des sciences Semlalia \\ BP S15 \\ Marrakech \\ Maroc \\ raouj@ucam.ac.ma
}

\author{
André Stef \& Gérald Tenenbaum \\ Institut Élie Cartan \\ Université Henri Poincaré-Nancy 1 \\ BP 239 \\ 54506 Vandœuvre Cedex \\ France \\ andre.stef@iecn.u-nancy.fr \\ gerald.tenenbaum@iecn.u-nancy.fr
}

